\title{
Choroid Plexus Blood-CSF Barrier: Major Player in Brain Disease Modeling and Neuromedicine
}

\author{
Conrad E. Johanson'*, Nancy L. Johanson ${ }^{2}$
}

'Department of Neurosurgery, Alpert Medical School at Brown University, Providence, RI, 02903 USA

${ }^{2} 408$ Autumn Trail, Georgetown, TX 78626, USA

Article Info

\section{Article Notes}

Received: June 11, 2018

Accepted: July 23, 2018

\section{${ }^{*}$ Correspondence}

Dr. Conrad Johanson, Ph.D., Professor Emeritus of Neurosurgery, Brown University Medical School, Providence,

RI, USA; Telephone No: 401-688-5299;

E-mail: conrad_johanson@brown.edu

(c) 2018 Johanson C. This article is distributed under the terms of the Creative Commons Attribution 4.0 International License

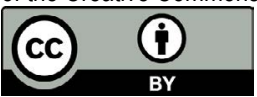

\section{ABSTRACT}

The choroid plexus (CP) of the blood-cerebrospinal fluid barrier (BCSFB) impacts CSF homeostasis, brain diseases and neuromedical translation. CP executes neuroendocrine, excretory, and neuroimmune actions. BCSFB diversely manages brain-spinal cord fluid environments, giving rise to a wide pathophysiology spectrum. Newly-discovered choroidal phenomena include integration of circadian clock signals, immune interaction with gut microbiotica, and expression of receptors to taste CSF composition. BCSFB tight junctions and transcellular mechanisms differ from blood-brain barrier (BBB) counterparts, variably regulating pathogen and leukocyte access to the CSF-brain nexus. This review highlights microbial agents, substrates and autoantigens using $\mathrm{CP}$ epithelial membranes to penetrate CSF and periventricular regions. Lipopolysaccharide (LPS) is analyzed as a barrier-damaging agent and neuroinflammation promoter. Transducing LPSand toll-like receptor activity produce CP-CSF cytokines in sickness behavior and virulent sepsis-associated encephalopathy. Agents/systems that counter oxidative activity such as matrix metalloproteinase 8 inhibitors and Nrf2 activators (bile acids and isothiocyanates) show promise as neural and CP protectants. One review theme emphasizes CP's preponderant role in initiating central diseases, and their remediation. In view of BCSFB permeativity alterations and epithelial transformation, we discuss: systemic lupus erythematosus, N-methyl D-aspartateassociated autoencephalitis, helicobacter disruption of BCSFB, toll-like receptor 2 stimulation in CP (neuroinflammation), the CP gateway for trypanosomes, and APOE-linked cholesterol transport into CSF. Another section treats concurrent involvement of BCSFB-BBB alterations in helminthic meningitis, forebrain ischemia, acute hyperthermia, leptin resistance/obesity, diabetes mellitus, and Alzheimertype neurodegeneration. Barrier impairment is analyzed by injury type, time course, therapeutic strategies, and translational neuromedicine principles. The restorative power of BCSFB-transported growth factors, hormones and medicinal agents is emphasized for strengthening CP-CSF homeostatic mechanisms in seizures, stroke and Parkinsonism. A worthy therapeutic aim is to attenuate CNS disorders triggered by BCSFB malfunction, using CSF-delivered therapeutic agents for promoting neural viability.

Introduction: Role of Barrier-Interfaces in Maintaining Brain Viability

Neuronal networks require stable extracellular fluid (ECF). Neurotransmission and electrical signaling depend on ECF of specialized composition. Transport/barrier/secretory systems at the blood-brain barrier (BBB) and blood-cerebrospinal fluid barrier (BCSFB) primarily regulate ECF. Choroid plexus (CP) is the main locus of the BCSFB, but circumventricular organs/meninges also contribute. There has been a tendency to underestimate BCSFB importance in favor of BBB. The two 
systems, however, are usually coordinated. Both barriers contain elements of the innate immune system and secrete messages (cytokine signals) into brain and CSF.

The BBB and BCSFB provide countless substrates via regulated carrier transport to the CNS (Fig. 1). Both are 'guardians', acting as barriers to thwart diffusion of potentially injurious molecules. More properly, CNS barrier systems function as 'barrier-interfaces' because of great solute exchange along with their barrier (impermeability) function. Thus, the dual BBB and BCSFB interfaces display dynamic transport physiology as a cardinal feature.

In mediating ECF homeostasis, CNS transport interfaces use intricate mechanisms. Tight junction components manifest as heterogeneous endothelial and epithelial membranes. Cerebral capillaries utilize a distinct array of endothelial transporters ${ }^{1}$. Moreover, CP contains a unique profile of transport and fluid-secretory systems ${ }^{2,3}$; see also Fig. 1. BCSFB and BBB provide the requisite substances via ECF dynamics to invigorate cerebral/spinal functions.

Brain microvessels chiefly translocate amino and fatty acids as well as glucose into parenchyma. CP specializes in secreting numerous trophic/stabilizing proteins ${ }^{4}$, e.g., brain-derived neurotrophic factor ${ }^{5}$, transthyretin,

\section{MAJOR TRANSPORT INTERFACES in CNS}

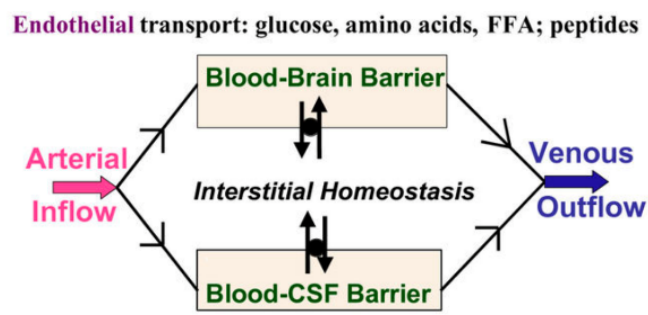

Epithelial transport: growth factors; vitamins B \& C; hormones

$$
\begin{gathered}
\text { Bidirectional Transport at Barriers: } \\
\text { Inward Secretion \& Outward Reabsorption }
\end{gathered}
$$

Figure 1: Cerebral vessels perfuse the neural/glial parenchyma (BBB) and choroid plexuses (BCSFB) suspended in four ventricles. Capillary wall endothelium contains inwardly-directed transporters that deliver mainly glucose, amino acids and free fatty acids (FFA) to nearby neurons. Choroid epithelial cells are specialized for transporting hormones, growth factors and neurotrophins into CSF for delivery to brain. The BCSFB barrier also secretes vitamin micronutrients and brain-modulating proteins: transthyretin and cystatin $C$ protease inhibitor. Upon entering ventricular CSF, these substances flow by volume transmission to spinal cord and brain, including Virchow-Robin spaces in cortical subarachnoid space. Not depicted is transfer of leukocytes and cytokines into CSF, accelerated in disease, traumatic injury and infections. Reabsorptive transporters for $K$ and organic anions remove excesses from ECF (capillaries) and CSF (CP). BBB and BCSFB work in concert to maintain ECF stability in health, and restore homeostatic balance following CNS insults. hormones and micronutrient vitamins. Choroidal and cerebral endothelial secretions physically combine, especially near brain parenchyma, to form the ECF milieu for neurons.

CSF movement thoroughly mixes all fluids in the CNS. CSF contacts virtually every barrier interface. CP primarily generates CSF that flows down the ventricles to the basal cisterns in the subarachnoid space (SAS); from there, CSF distributes widely to far reaches of the brain/cord (Fig. 2). Multi-regional transport activities and CSF flow create an ECF blend ('hybrid') of BBB and CP secretions ${ }^{6}$. Fluid blending is facilitated by the paravascular system that starts as SAS CSF entering the superficial cortex at the VirchowRobin spaces (Fig. 2). Overall, then, the driving force that propels CSF forward into the ventricular, subarachnoid and paravascular systems originates largely at the brisklyperfused and secreting CP villi.

Each CP villus contains a rich, inner vascular core, and adjacent interstitium. Both are surrounded by a ring of epithelial cells in adults (Fig. 3). The rapidly-metabolizing epithelium is highly equipped with organelles (Fig. 4). Abundant endoplasmic reticulum, golgi apparatus and mitochondria enable profuse synthesis of proteins and peptides (Tables 1 and 2). Upon secretion into CSF, these choroidally-derived proteins/peptides flow through the ventriculo-SAS to neuronal and glial targets. Targeted regions ${ }^{6,7}$ are both proximate to and distant from CP tissues. Upregulated BCSFB secretions into CSF-ECF abet healing of damaged neurons.

Choroid Plexus and BBB Disruption: Extracellular Dyshomeostasis Places Neurons at Risk

The first sequential casualty of barrier injuries is the nearby ECF composition. Distorted ECF biochemistry disables neuronal/synaptic functions. This disablement is proportional to transporter breakdown and induced leakiness. Before discussing specific disorders/diseases that destabilize the barriers, we lay out the general features of i) barrier physiology: transporters, solute gradients across barriers, and mitochondrial energetics, and ii) barrier pathophysiology: cytokine disruption of barrier cells, innate immune response, and neuroinflammation. The immediately-following paragraphs overview general physiologic principles, together with models and experimental tools associated with barrier studies, as a prelude to the later treatment of specific barrier injuries/ pathologies.

\section{Barrier transport/energetics phenomena}

The complexity of solute transporters and channels in barrier cells, and their regulation by receptor and enzymatic activities, cannot be overstated. It is beyond the present scope to detail the myriad of transporters at the BCSFB 
Table 1. Newly appreciated and established functions/actions at the choroidal blood-cerebrospinal fluid interface

- Integrator of circadian clock periodicity signals, generated in the choroidal syncytium and conveyed to the suprachiasmatic pacemaker in the hypothalamus

- Source of stem cells and progenitor elements, putatively for the reconstitution of CP epithelium and periventricular regions, following injuries such as ischemia and trauma

- Relay station for plasma hormone signals that, once secreted into the ventricles, reach the hypothalamus for hormonal regulatory phenomena, e.g., feedback inhibition

- Gateway through which microbes (e.g., bacteria, viruses and trypansomes) from intestine (and other regions) invade the blood, and then distribute to and injure the choroid plexus-CSF system

- Interface for immunologic molecules and cells to access the epithelium, thereby generating cytokine signals and activating leukocyte transfer to the CSF-periventricular nexus

- Liver-type actions to metabolize/detoxify drugs and xenobiotic substances that could harm the brain

- Kidney-type organ that purifies/buffers extracellular fluid composition (e.g., $\mathrm{H}$ and $\mathrm{K}$ ions) for neuronal networks

- Receiver of sex hormone signals (e.g., progesterone) to regulate choroid plexus functions, e.g., transthyretin synthesis/secretion for brain protein modulation

- Provider of polypeptides and proteins (e.g., cystatin C) for regulation of cerebral metabolism, and for mediating restoration of brain injury

- Sentinel-like function to detect neuroinflammation and injury-generated molecules in brain

- Intracranial pressure-regulating organ that responds to elevated ventricular pressure by hormonally (e.g., atrial natriuretic peptide) downregulating CSF formation rate

- Source of the CSF that provides the watery medium for volume distribution of molecules within the ventricles and to cortical subarachnoid regions (for entry into paravascular flow routes)

- Taste receptor sensing of CSF bitter/toxic compounds, hypothetically for homeostatically refining the chemical composition of brain extracellular fluid milieu

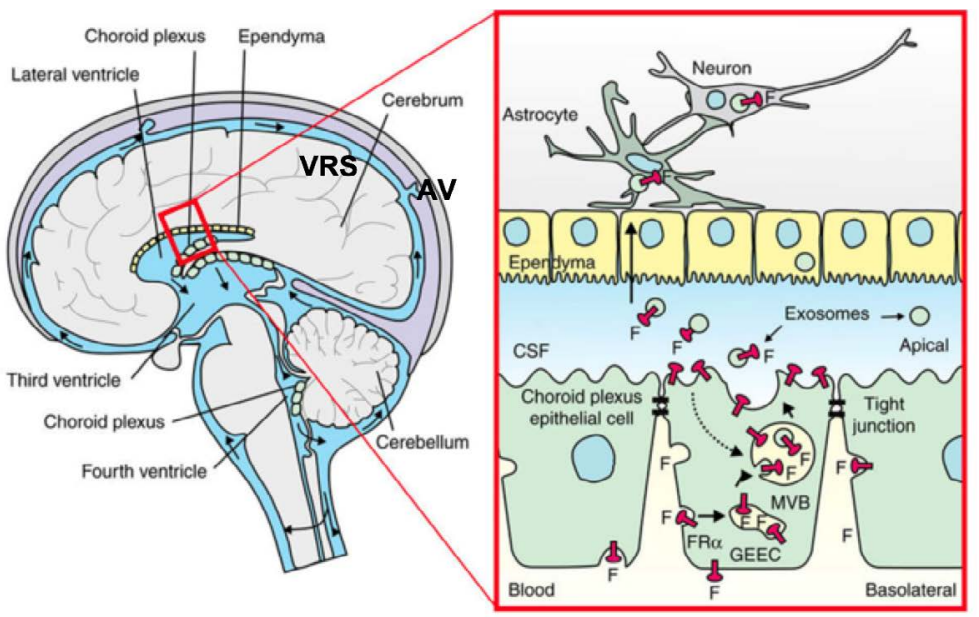

Figure 2: CSF formed by CP cells (green) flows down the ventricular system into subarachnoid space (SAS) surrounding cortex, cerebellum, brain stem and spinal cord. Ependymal cells (yellow) line the ventricle walls, providing a permeable interface between CSF and brain ECF. Astrocyte processes extend to ependyma and to capillary walls of BBB. CSF oscillates gently to-and-fro with cardio-respiratory rhythms while undergoing bulk flow (arrows) to distal reaches of SAS. CSF under pressure flows over the cortices to drain across arachnoid villi (AV) into venous blood (lavender), or to enter the brain's paravascular system at the Virchow-Robin spaces (VRS). Bottom right: Exosome vesicles, containing folate, $\mathrm{F}$ (red), are released into CSF for transit to various parenchymal targets. MVB, multi-vesicular bodies GEEC, early endosomal compartments Adapted from ref. 44 (Grapp et al.)

and $\mathrm{BBB}$, but the reader can find comprehensive treatises elsewhere ${ }^{7-10}$. Active Na distribution across membranes determines the fluid balancing between extracellular and intracellular compartments. It is timely to assess $\mathrm{Na}$ transporters that couple to $\mathrm{H}_{2} \mathrm{O}$ movement. For the BCSFB and BBB, the Na pump (Na-K-ATPase) faces the CNS side of the barrier and establishes steep Na gradients across the low-[Na] barrier cells. Vigorous Na pumping is critical for cell volume, voltage gradients, and the net trans-barrier movement of organic solutes. Another major mechanism is the Na-K-Cl cotransporter, that promotes fluid movement and contributes to barrier cell $[\mathrm{Cl}]$ stability and CSF-ECF $\mathrm{K}$ homeostasis. A key to understanding cerebral and $\mathrm{CP}$ edema (e.g., in stroke, trauma and hyperthermia) is that fluid retention stems from compromised $\mathrm{Na}$ transport and reduced water removal. Conversely, $\mathrm{Na}-\mathrm{H}_{2} \mathrm{O}$ fluid movements at the barriers may increase in certain inflammatory states. 


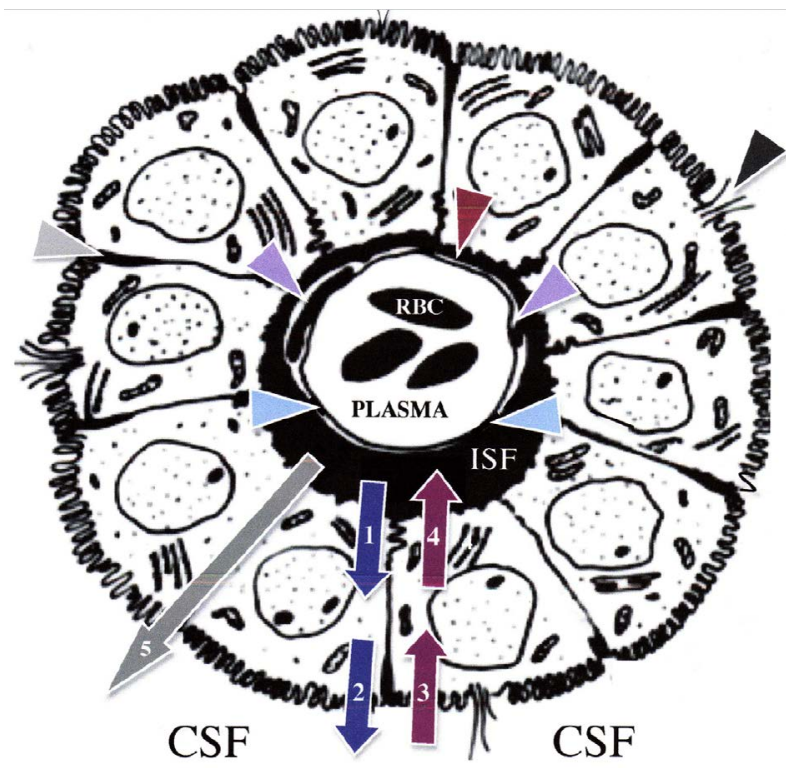

Figure 3: CP tissues have multiple villi projecting like a fishnet into ventricular CSF. This schema is reconstructed from micrographs of intact CP. Several epithelial cells (see Fig. 4 for ultrastructure) surround each villus. Each CSF-floating villus receives brisk blood flow from choroidal arteries. Plasma filters across highly-permeable capillary walls to the interstitial fluid (ISF) compartment ( 15\% of tissue volume). ISF contains a matrix where cytokines/leukocytes reside. Matrix interacts with the basal (ISF-touching) epithelial plasma membrane. The innermost capillary (with red blood cells, RBC) has a fenestrated permeable endothelial membrane (red arrowhead) and leaky inter-endothelial junctions (blue arrowheads). The underlying basement membrane of capillaries abuts extracellular matrix. Nucleated endothelial cells are shown as lavender arrowheads. Regulated solute traffic across epithelial cells is active (energy requiring) and dynamic. Inward movement of plasma solutes (secretion) occurs transcellularly across epithelial cells, by a 2-step sequential process: arrow \#1, uptake across basolateral membrane; and \#2, extrusion across apical membrane. Outward movement (reabsorption) of CSF solutes transcellularly is also a 2-step process, by apical membrane uptake (arrow \#3) from CSF, followed by basolateral release (\#4) into choroidal interstitium, then plasma. Minimal diffusion of substances (due to resistance of tight junctions, gray arrowhead) occurs paracellularly between adjacent epithelial cells (arrow \#5). Cilia (black arrowhead) promote mixing/movement of CSF. From reference \#68.

Quantifying solute gradients across membranes that demarcate compartments (plasma, brain ECF, CSF) importantly assesses barrier integrity and CSF neurochemistry. The CSF/plasma ratio for substances informs on CSF viability and neural disease progression. Healthy individuals display characteristic values for the $\mathrm{CSF} /$ plasma ratio ${ }^{11}$. When the CNS is insulted by injuries, the $\mathrm{CSF} /$ plasma ratios for solutes deviate from normality. $\mathrm{CSF} /$ plasma for the diffusible albumin (mw 68,000) and urea (mw 60) increase upon barrier breaching, as in mild cognitive impairment and Alzheimer's disease ${ }^{12}$. Organic substrates such as creatine/creatinine, actively transported, also undergo a major change in CSF/plasma values in barrier-harming diseases ${ }^{12}$. When $\mathrm{CP}$ and $\mathrm{BBB}$ are not adequately perfused, as in neurodegeneration and other energy-deprivation states, the CSF/plasma ratios of solutes trend towards 1.0 (i.e., an equilibrative passive dispersal, rather than a steady-state distribution set up by ATP-fueled transport systems).

$\mathrm{Na}$ transport to maintain central fluid dynamics and transcompartmental gradients depends on mitochondrial ATP availability. CP epithelium and brain endothelium abound in mitochondria. Choroid mitochondrial ATP energizes active transport to set up the transmembrane $\mathrm{Na}$ gradients that drive CSF formation. Mitochondrial failure, primary by gene mutation ${ }^{13}$ or secondary to amyloid-beta (A $\beta$ ) accumulation in CP as in Alzheimer's disease, curtails CSF dynamics and disrupts solute homeostasis/gradients; cell energy failure from ATP diminution also enhances oxidative stress. Mitochondrial apoptosis, associated with disordered Sirtuin 1 gene function, increases in barrier injury and autoimmune disease ${ }^{14}$. The mitochondrial division inhibitor, Mdivi-1, suppresses apoptosis and protects against barrier breakdown ${ }^{15}$. Deficient mitochondrial (ATP-generating) function diminishes organic solute movements in $\mathrm{CP}$, resulting in lower folate and higher homovanillic acid concentrations in $\mathrm{CSF}^{16}$. Accordingly, correcting CSF-brain dyshomeostasis in neural disorders should include stabilizing mitochondrial function and energy state in barrier epithelium and endothelium.

\section{Barrier alteration by inflammatory cytokines: triggering of neuroinflammation}

In addition to the aforementioned bioenergetic deficiency problems, systemic inflammation pathophysiology also threatens the wellbeing of BBBBCSFB. Barriers are inflamed by vascular disorders (stroke), traumatic brain injury, infections and byproducts 


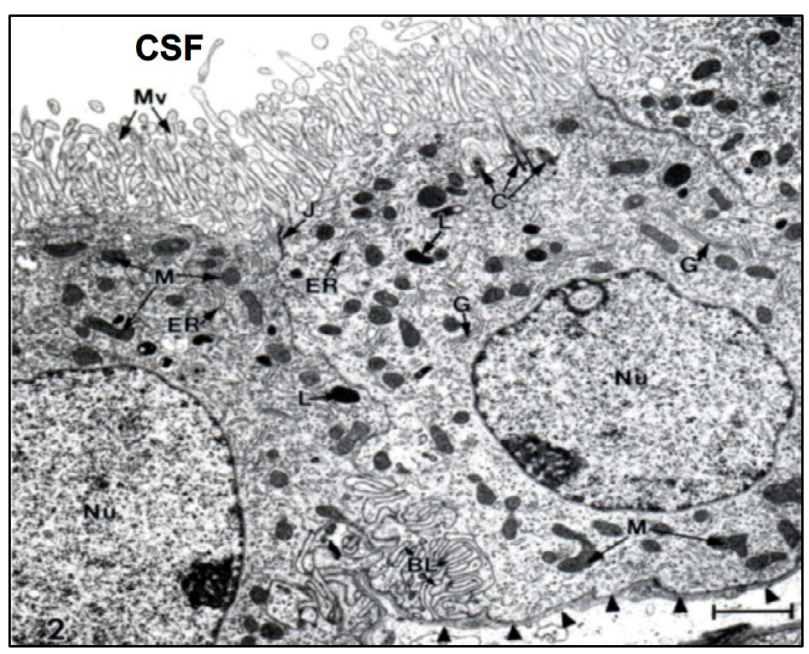

Figure 4: Tight junctions $(\mathrm{J})$ weld adjacent choroid cells at their CSF-touching loci. Narrow paracellular pathways, just under the tight junctions, finely separate adjacent cell lateral membranes. Extensive microvilli (Mv) on apical membrane confer extensive surface area for transport processes. Abundant mitochondria (M), endoplasmic reticulum (ER) and Golgi apparatus (G) support extensive protein synthesis and solute transport, and energize high rates of metabolism and CSF formation. The basal labyrinth (BL) maximizes transport area by intertwining apposing membranes of adjacent choroid epithelial cells. C, centrioles $\mathrm{Nu}$, nucleus Adult rat lateral ventricle $\mathrm{CP}$ was fixed with $\mathrm{OsO}_{4}$ for electron microscopy. Scale bar (bottom, right) $=2 \mu \mathrm{m}$

of neurodegenerative disease, e.g., amyloid plaque ${ }^{17}$. Disrupted transport interfaces provoke ECF biochemical instability and immune-brain imbalance in parenchyma of cortical, CSF and periventricular regions ${ }^{18-22}$. Lipopolysaccharide (LPS), used in many experimental models of neurodegeneration, disrupts the BBB and BCSFB; consequently, upon penetrating the damaged barriers, LPS drives the NF-kB associated progressive inflammatory signaling in the parenchyma. This is a prominent feature of neurodegeneration.

Perturbed immune-brain interaction underlies many neurological/psychiatric disorders: status epilepticus, stroke/ischemia, and the neurodegenerative Parkinson's and Alzheimer's diseases. Neurons malfunction when overrun with ECF perturbations. To heal both the barrier elements and parenchymal cells, the BBB and BCSFB in injury states compensatorily secrete peptides, growth factors and neurotrophins ${ }^{18,23,24}$. These beneficial factors stabilize extracellular-intracellular fluid balance for neural health ${ }^{25}$. Various modulating factors, presented in Table 2, are addressed in topics below.

Several brain disorders discussed in later sections are caused/exacerbated by disrupted barriers that encompass neuronal networks. Frequently, barrier opening precedes clinical symptoms. Barrier breaching enables central invasion by foreign substances, microbial agents and
Table 2. Choroid plexus hormones, neurotrophins, and growth factors:

transported/secreted into CSF to support BCSFB and multiple brain regions

Hormones transported/distributed by CP from blood to CSF

Leptin, an appetite and metabolism regulator

Prolactin, a reproductive hormone and lactotroph

Adipsin and adiponectin, fat hormones that regulate neuroinflammation

Orexin A or hypocretin-1, an arousal and appetite hormone

Neurotrophins/neurotrophic factors synthesized/secreted by CP into CSF

Brain derived neuronal factor (BDNF)

Glial derived neuronal factor (GDNF)

Neurotrophin-3 (NT-3)

Neurotrophin-4 (NT-4)

Ciliary neurotrophic factor (CNTF)

Growth factors transported/distributed by CP from blood to CSF Insulin-like growth factor-1 (IGF-1)

Hormones synthesized/secreted by CP into CSF

Arginine vasopressin (AVP) fluid regulator

Augurin, an ecrg4 product that regulates mitosis

Hepicidin, an iron regulator

\section{Growth factors synthesized/secreted by CP}

Nerve growth factor (NGF)

Basic fibroblast growth factor-1 (FGF-1)

Acidic fibroblast growth factor-1 (FGF-2)

Transforming growth factor beta (TGF $\beta 1 ; \beta 2 ; \beta 3)$

and TGF $\beta$ latent binding proteins (LTBP-1 and -3 )

Hepatocyte growth factor (HGF)

Insulin-like growth factor-2 (IGF-2) and binding protein

Vascular endothelial growth factor (VEGF) and VEGFC ligand

Granulocyte colony-stimulating factor (G-CSF), an hematopoietic factor

Amphiregulin, a member of epidermal growth factor family

immune elements. Consequently, ECF composition is tainted by the centrally-invading plasma proteins and immune elements normally screened by barriers. Sentinellike detection and resolution of neuroinflammation depend on an intact $\mathrm{CP}$ and neurovascular unit.

Seizures stem from BBB opening in cerebral tumors, trauma and stroke ${ }^{26}$. BCSFB permeability increases after stroke or mechanical injury, predisposing to seizures. Stabilizing the compromised CNS barriers, to suppress neuroinflammatory progression and toxic immune reactions ${ }^{27}$, significantly restores neuronal excitability and thwarts seizures. An electroconvulsive model reveals upregulated growth factors, including erythropoietin, and trophic proteins in CP responding to seizures ${ }^{28}$. Hormonal erythropoietin exogenously administered (or induced in CP) holds promise for augmenting BCSFB regulators ${ }^{29}$ to foster CP-CSF-brain homeostasis. Such positive actions by erythropoietin and other trophins reduce CSF oxidants, attenuate neuroinflammation, and lower depression as well as seizure activity. 
Cells of the BCSFB and BBB secrete neurotrophins, immunoregulators (interferons), and anti-inflammatory cytokines for CSF adaptation to circulating vascular stressors. Accordingly, the brain endothelium and choroidal epithelium 'fight back' against the systemic inflammation that immunologically irritates transport interfaces. Severe systemic inflammation threatens BCSFB and BBB integrity as barriers become greatly permeabilized with dire consequences for brain. Peripheral inflammation shifts the ECF to a pro-inflammatory state. A popular method to create a pro-inflammatory environment in the CNS is by injecting the bacterial LPS peripherally so that LPS/TLR4 receptors at the BBB-BCSFB are stimulated to produce 'inflammatory responses' within the barrier elements. This local inflammatory response, i.e., cytokine production in barrier cells, is then transmitted outward into brain ECF and CSF.

BBB injury from cytokine upregulation accompanies most brain diseases/infections. LPS 'challenge' has been useful for experimentally 'dissecting' components of inflammatory damage to brain microvessels. Plasma cytokines/microbial products, via toll-like receptor stimulation, transduce barrier targets to express immunoregulatory molecules for secretion. There are multiple reports of LPS consistently increasing BBB permeability and vesicular activity, and harming cerebral endothelium; adverse effects on glycocalyx, glia limitans and astrocytes also eventuate from LPS administration. In disease (vs. health) the BBB is more sensitive to systemic inflammation ${ }^{30}$.

LPS disruption of BBB permeability is variable, depending on LPS dose, regional effects and neurochemical factors. LPS-induced global BBB breakdown is less severe than that by focal cold injury ${ }^{31}$. Banks et al ${ }^{32}$ demonstrated that LPS enhances BBB leakiness to ${ }^{14} \mathrm{C}$-sucrose and albumin in several regions, but not in hypothalamus even at a very high dose. Pathophysiologic state matters for LPS actions. LPS intensifies BBB opening in animals with acute liver failure $^{33}$. Interestingly, synuclein expression increases the BBB 'leakiness response' to LPS stimulation ${ }^{34}$. Clearly, LPS compromises BBB integrity, an effect amplified by various diseases. The anti-oxidant melatonin experimentally counters LPS-induced damage to $\mathrm{BBB}$ and $\mathrm{CP}^{35}$.

The BCSFB also responds sensitively to circulating LPS. Over $400 \mathrm{CP}$ genes are differentially expressed following LPS injection of mice ${ }^{36}$; upregulation clusters into immunoregulating and extracellular matrix-remodeling genes, while downregulation results in less control of barrier maintenance. This transcriptome alteration centers around the so-called acute-phase response to peripheral inflammation. CP is ideally situated, with the help of CSF which carries the secreted proteins ${ }^{37}$, to pass immunosignaling molecules into the ventricle-brain axis.
In the relatively weak neuroimmune response of 'sickness behavior', cells at the CNS-immune interface (CP epithelial/stromal cells, leptomeninges, and hippocampal neurovascular cells) are 'initial responders' to LPS injected into mice ${ }^{38}$. Thus, in sickness behavior, cytokine production in barrier cells ( $4 \mathrm{hr}$ post-LPS endotoxemia) precedes the spread of barrier-generated immune signals by ECF. Later, responding to barrier-produced and -distributed cytokines, hippocampal astrocytes secrete cytokines that expand the CNS immune reaction to the original source of peripheral endotoxin ${ }^{38}$.

A more virulent disorder than sickness behavior is the sepsis-associated encephalopathy (SAE). SAE varies in pathologic severity but does not necessarily involve CNS bacterial invasion; this disorder carries high morbidity/ mortality and is characterized by neuroinflammation, circulatory dysfunctions, CNS barrier breakdown and cognitive impairments ${ }^{39}$. Long-term BBB dysfunction (MRI evaluation) augments cerebral metabolite distortions and modifies neurobehavior in the LPS-induced SAE model $^{40}$. BCSFB is also hit hard by experimental sepsis, engendered by either LPS i.p. or cecal ligation/puncture; upregulated matrix metalloproteinase 8 (MMP8) is a key factor in disrupting CP extracellular matrix and epithelial structure $^{41}$. The greatly compromised BCSFB significantly contributes to the consequent development of damaging neuroinflammation. Due to potential lethality, the systemic inflammatory response that destroys CNSbarriers is a sepsis research area urgently in need of pharmacotherapeutic remedies. Nanobody agents that counter sepsis-induced MMP8 activity ${ }^{42}$ particularly for BCSFB application, may help minimize sepsis/inflammatory damage to the CP-CSFbrain nexus.

LPS continues to be useful in stimulating/characterizing $\mathrm{CP}$ immune phenomena. That is, bacterial molecules stimulate $\mathrm{CP}$ epithelial receptors, generating immunoproducts and vesicles for transfer into CNS. Low-grade systemic infection or LPS/TNF $\alpha$ administration prompts $\mathrm{CP}$ to form vesicles (exosomes) containing small RNA molecules ${ }^{43}$. CP discharges these vesicles into $\operatorname{CSF}^{44}$, presumably for regulating astroglial/microglial targets (Fig. 2). CSF bulk flow promotes vesicle distribution throughout brain and spinal cord. Vesicle dynamics also raises therapeutic possibilities for improving CP-toneuron communication (signaling). Barrier-generated vesicles, their composition and distribution kinetics, deserve further investigation. Exosome inhibition of $\mathrm{CP}$ vesicle release has potential application for controlling neuroinflammation ${ }^{45}$. Pharmacologic use of CSF-borne vesicles as non-immunogenic drug (and RNA) carriers holds promise ${ }^{46}$ for next generation cell-biological repair of the dysregulated cerebral metabolism and neuroimmunity in neurodegeneration. 
The sections below expand upon previously integrated CP-BBB data ${ }^{47}$, by updating disordered homeostatic mechanisms in the CSF-brain nexus. For wide-ranging pathophysiologies, the relative roles of each barrier are examined. We emphasize that in certain disorders the $\mathrm{CP}$ is the main barrier affected. In others, BBB also fails. A recurring theme is that brain recovery requires reinstated dual-barrier stability for strong ECF homeostasis.

\section{Separate vs. Overlapping Barrier Functions}

Stabilizing functions work jointly, but sometimes separately by BBB or BCSFB. Cerebral capillaries and choroid plexuses remove from extracellular fluid excesses of various solutes. These include clearing superfluous $\mathrm{K}$, inorganic anions (e.g., iodide and thiocyanate) and organic anions (e.g., 5-hydroxyindole acetic acid and homovanillic acid catabolites of biogenic amines). Certain peptides are reabsorbed into blood by BBB or BCSFB, but there are regional differences in transporter expression. Thus, the Pept2 transporter in apical membrane of CP (but not $\mathrm{BBB}$ ) removes small peptides from CSF, e.g., the dipeptide alanine-alanine ${ }^{48}$. Transport analysis of the disaccharide glycylsarcosine in isolated mouse $\mathrm{CP}$ in which the pept2 gene was completely ablated reveals Pept2 as the main member of the peptide transporter family that reabsorbs dipeptides at the $\mathrm{BCSFB}^{49}$.

Other efflux systems, such as the multidrug resistance protein (MRP), organic anion transporter (OAT), and organic anion transporting polypeptide (OATP), are present in both $\mathrm{BBB}$ and $\mathrm{BCSFB}^{48}$. These clearance transporters remove organic anions from brain ECF and CSF. Cephadroxil, an antibiotic, is avidly removed from central ECF by MRP, OAT and OATP. Such removal from brain-CSF by these transporters may lower the agent concentration to sub-therapeutic levels in the CSF. Preventing undesired reabsorption, i.e., efflux from CNS, comes about with competitively-inhibiting drugs such as probenecid. Neurodegeneration and neuroinflammation disrupt CNS barrier transporters. Such barrier disruption adversely affects ECF homeostasis, disease progression and neural pharmacotherapy ${ }^{50}$.

On the CSF influx side, several endogenous compounds penetrate substantially by the BCSFB. CP acts as a 'relay station' in transporting hormones from blood to hypothalamus. These include prolactin and insulin-like growth factor 1 secretion, from plasma-to-CP-to-CSF. Hormones then flow by volume transmission (Fig. 5) to hypothalamic target cells ${ }^{51}$. Vitamin $\mathrm{C}$ is transported into CNS via the ascorbate SVCT2 transporter in CP epithelium $^{52}$, but not BBB endothelium; accordingly, SVCT2 knockout at the BCSFB severely depletes brain ascorbate ${ }^{53}$. Major transport of folate across BCSFB is essential for proper neural tube development; conditions associated

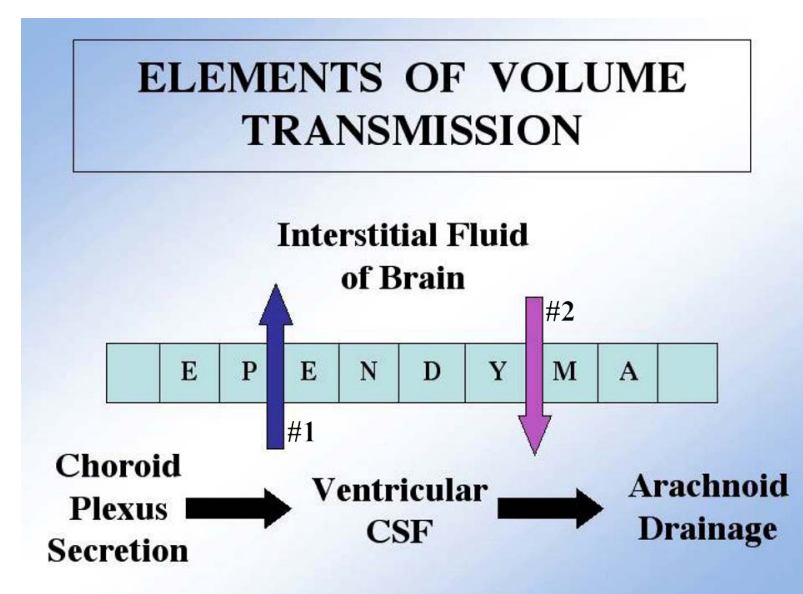

Figure 5: CSF volume transmission is a bulk flow system, originating as CP secretion, that distributes solutes and cells (e.g., leukocytes) throughout the ventriculo-subarachnoid spaces (SAS). CSF streams widely throughout the CNS. Prior to draining at distal arachnoidal sites into venous-lymphatic fluids, the CSF freely exchanges solutes between ventricles/SAS and brain interstitial (extracellular) fluid. Solutes such as vitamin C, transthyretin, cystatin $\mathrm{C}$ and leptin (from CP) diffuse into brain across (arrow \#1) the permeable ependyma. Simultaneously, substances such as superfluous proteins, amino acids, $\mathrm{K}$ and catabolite organic anions, diffuse (arrow \#2) from brain into CSF for clearance. Acting dually as a supply and excretory (sink) system, CSF volume transmission is a continually-flowing watery medium mediating solute/cell distribution along extracellular pathways. Endocrine signaling (hormone transit, CP to hypothalamus) is enabled by CSF streaming. CSF volume transmission ends when SAS fluid flows into venous and lymphatic channels. Adapted from Johanson et al., CSF Research 2004; 1:3.

with marked CSF folate dwindling lead to spina bifida/ hydrocephalus. Transthyretin, secreted by CP epithelium but not cerebral endothelium, facilitates thyroid hormone transport into CSF-CNS and protects neurons by stabilizing amyloid conformation ${ }^{54}$. Aquaporin 1 (AQP1) at the BCSFB facilitates water penetration into $\mathrm{CSF}^{55}$. AQP1 is not normally in the BBB, which instead expresses AQP4 in astrocyte feet to enhance water fluxes between plasma and brain.

Organic metabolites, e.g., creatinine/urea, and cytokines interact with both barriers ${ }^{56,57}$. Certain immune phenomena, e.g., leukocyte migration, may associate more prominently with BCSFB than BBB. Under particular conditions, the $\mathrm{CP}$ gate allows leukocyte penetration to downstream periventricular regions ${ }^{58}$. This occurs in multiple sclerosis relapse. Viruses, bacteria and fungi exploit CP as a CSF 'port of entry' into brain ${ }^{59,60}$. HIV uses CP interstitial milieu (Fig. 3) to promote evolution of drugresistant viral strains ${ }^{61}$, with untoward consequences for CSF that contacts multiple CNS regions.

Proteins leak from blood into brain and CSF. This 'proteinaceous' infiltration increases in aging ${ }^{62}$ and 
neurodegeneration. Proteins diffuse across BCSFB more extensively than $\mathrm{BBB}$, due to more permeable tight junctions in $\mathrm{CP}^{63}$ than cortical capillaries. Accordingly, in neural diseases the CSF-to-serum ratios of proteins such as albumin (mw $\sim 68,000$ ) primarily reflect BCSFB breaching ${ }^{64}$. This concept ${ }^{65}$ needs to replace the established inaccurate notion that the CSF-to-serum ratio of proteins mainly assesses brain microvessel permeability.

Our review highlights the spawning of new concepts on regulated interfaces separating blood, CSF and brain. Multi-faceted regulators at $\mathrm{CP}$ epithelium and cerebral microvessels safeguard neuronal milieu. Many polypeptides and proteins, arising from BCSFB transport into $\mathrm{CSF}^{66}$, modulate brain viability. Synaptic environments suffer upon failure of homeostatic transport mechanisms at the barriers. Hence, following severely-perturbed CSFbrain homeostasis, neurotransmission falters. Cognitive and behavioral losses ensue. Extensive barrier trauma leads to morbidity crises and even death. Therefore, novel strategies are sought to safeguard barrier systems or quickly restore their function.

A continuing concern is the BBB's role in CNS impairment. Much focus is on neurovascular unit failure in trauma, ischemia and neurodegeneration ${ }^{67}$. We now integrate data primarily for BCSFB damage, but also for concurrent breakdowns of $\mathrm{CP}$ and $\mathrm{BBB}$. Our treatment covers new stratagems with CP-CSF pharmacology to restore compromised barriers.

\section{Historical vs. Current Paradigms of Barrier Investigations}

Past research overstated BBB importance and obscured the BCSFB role. A PubMed search in May 2018 revealed $\sim 44,000$ articles for BBB and $\sim 10,000$ for BCSFB/CP. Attention directed to BCSFB malfunctions prompts novel possibilities for translational research ${ }^{25}$. In reality, both systems normally operate together. Their actions therefore should be integrated in models of neural homeostasis and disease ${ }^{47}$.

Brain is broadly immersed in CSF, and CP forms most of the $\mathrm{CSF}^{68}$. It follows that the villous CP plays a chief role in central homeostasis (Fig. 2). CP floats in ventricular CSF, spreading out like a fishnet. This maximizes surface area for microvilli exchange (Fig. 3). To-and-fro movement of CSF, responding to cardiac and respiratory pulsations, physically displaces $\mathrm{CP}$ villi. This allows thorough mixing of CSF for monitoring and adjustments. Currently undergoing revision is the historically inaccurate perception of $\mathrm{CP}$ as a 'minor player' due to small surface area. BCSFB is currently regarded as a highly dynamic transport interface with activity intensity on par with $\mathrm{BBB}^{68}$. This notion is bolstered by findings that CP-generated net turnover of CSF greatly exceeds BBB fluid generation.
$\mathrm{CP}$ as a multi-functional organ has been stressed over recent decades. Its wide array of actions/reactions benefits the brain and spinal cord (Table 1). An interesting liverlike analogy is $\mathrm{CP}$ epithelial glutathione detoxification to protect neurons ${ }^{69}$. A kidney-like function is the newlydiscovered $\mathrm{Cl}^{-} / \mathrm{H}^{+}$exchanger in $\mathrm{CP}$ luminal membrane. $\mathrm{Cl}^{-} /$ $\mathrm{H}^{+}$exchange is being explored for CSF $\mathrm{pH}$ and acid-base regulation ${ }^{70}$. Multiple taste receptors are expressed in CP epithelial cells (Tas1r1, Tas1r2, Tas1r3, Tas2r109 and Tas2r144); transduction of their signals is consistent with a putative role of tasting bitter, noxious compounds in $\mathrm{CSF}^{71}$. The role of taste signaling at the BCSFB, possibly in regard to refining the neural milieu, awaits elucidation.

Newly-described choroidal functions stimulate thinking for future CP-CSF modeling. Recent findings on CP internal housekeeping includes syncytial actions that tie together, by gap junction communication ${ }^{72}$, periodicity signals affecting extra-choroidal regions. Physiologic rhythms mediated by the hormonally-regulated CP circadian clock $^{73}$ modulate, via CSF delivery of cytokine signals, the 'master coordinator' circadian clock in the hypothalamic suprachiasmatic nucleus ${ }^{74}$.

The prospect of using specific bile products, e.g., tauroursodeoxycholic acid, in neurological disease management is a captivating innovation on the horizon ${ }^{75}$. Stimulation of brain cell receptors by deoxycholic acid derivatives restores mitochondrial function and ATP energy levels. This protects against experimental neurodegeneration advancement in certain Parkinson models. Nrf2, short for nuclear factor-erythroid 2-p45 derived factor, is a master regulator protein that counters free radicals by stimulating several anti-oxidative enzyme systems. Upregulated transcription factor Nrf2, by tauroursodeoxycholic $\operatorname{acid}^{76}$ and isothiocyanates ${ }^{77}$, protects dopaminergic neurons ${ }^{78}$ and BCSFB against excessive oxidation (as in neurodegenerative stress). This Nrf2 protective effect is mediated by a rise in the anti-oxidant enzymes hemeoxygenase 1, glutathione peroxidase and quinine oxidoreductase ${ }^{77}$. Consequently, induced Nrf2 expression protects $\mathrm{CP}$ and dopaminergic neurons by preventing progressive cytotoxic damage. The isothiocyanates, sulforathane and allyl-isothiocyanate, from cruciferous vegetables also stimulate Nrf2 expression, thereby protecting CP from oxidative disruption ${ }^{77}$. Nrf2 augmentation by tauroursodeoxycholate and isothiocyanates is a putative pharmacological target to strengthen $\mathrm{CP}$ homeostatic reserve and nigral neuron viability.

Another recent, intriguing BCSFB discovery, involving peripheral organs, is bacterial infection interactions between $\mathrm{CP}$ and the gastro-intestinal tract $^{79}$. Gut inflammation leads to neurodegeneration putatively linked to BCSFB breaching. Such CP inflammation relates 
to gastric mucosal infection spread into the CNS. Thus, CPCSF functional interactions include peripheral phenomena as well as intra-brain homeostatic adjustments.

Now we elaborate structural/functional alterations of barriers that distort fluid parameters in neuronal and glial networks. First, we treat recently-reported phenomena transpiring mainly at CP. Thereafter we introduce new concepts on concurrent actions, beneficial and adverse, at the BCSFB and BBB. Evaluating the respective barrier roles in a range of pathologies reveals precise pharmacologic targets to reconstitute injured brain. Evidence is organized below advocating for an approach to target the BCSFB with medicinals that heal the epithelium damaged by microbes and other agents. A worthy therapeutic goal is to alleviate major CNS disorders triggered primarily by $\mathrm{CP}$ malfunction, as a substantial mechanism (apart from the BBB) to improve neural status compromised by CSF toxicity-triggered brain injury.

\section{Barrier-Interface Actions Localized Mainly to Choroid Plexus}

Strong evidence implicates CP-CSF-brain solute transport and distribution as critical for CNS ontogeny. Malfunctions of transport systems and CSF volume distribution (as in congenital hydrocephalus) predispose to developmental neural diseases/disorders. Investigators increasingly analyze both barriers simultaneously to devise integrative transport models. Such modeling will likely find application in neurotherapeutics. Below we localize the onset of neural disorders to BCSFB disablements. CP functional diversity, when disrupted, gives rise to a wide spectrum of CSF-brain pathophysiologies. Translational opportunities deserve exploration to counter impairments (discussed below) that selectively afflict CP interstitialepithelial zones.

\section{Systemic lupus erythematosus}

Neuropsychiatric systemic lupus erythematosus (NPSLE) is an autoimmune disease caused/exacerbated by leukocyte and immunoglobulin penetration into CSF, then to periventricular regions that become inflamed. Formerly, a leaky BBB was implicated in NPSLE onset; however, a paradigm shift in transport modeling may be emerging. Gelb and colleagues tested an SLE mouse model (MRL/lpr) for penetration of permeability probes: immunoglobulin G, dextran (mw 10,000), and horseradish peroxidase (HRP). These large-sized probes and lymphocytes did not penetrate BBB but readily crossed $C P$ via transcytosis (not tight junctions). Thus Gelb et al. challenge the disrupted BBB model of NPSLE, concluding that antibodies/lymphocytes permeate a perturbed BCSFB en route to inflamming the subependymal regions ${ }^{80}$. To explain NPSLE, they propose shifting the experimental focus to CP-CSF.

\section{N-methyl D-Aspartate-associated autoencephalitis}

Autoantibodies to N-methyl D-Aspartate (NMDA) receptors wreak havoc on neurotransmission in autoencephalitis. An imbalanced inflammatome promotes neuroinflammation-mediated cognitive decline. Autoantibodies against NMDA receptor are in CSF. Still, studies to attribute the autoantibody penetration centrally to the BBB have not been fruitful. Hammer et al. ${ }^{81}$, for example, found that intravenous NMDA receptor antibody did not penetrate a healthy BBB.

BCSFB is more permeable than BBB, especially in neurodegeneration. Thus, it makes sense to determine if $\mathrm{CP}$ in $\mathrm{MCI} /$ Alzheimer's disease allows enhanced CSF penetration of NMDA receptor antibodies. Recent verification of augmented BCSFB permeability to NMDA (elevated CSF/serum albumin) also found increased NMDA receptor autoantibody in $\mathrm{CSF}^{82}$. The Busse et al. findings reveal that certain patients transmit more NMDA receptor antibodies into CSF as neurodegeneration worsens. This subset points to a dual problem: a) the compromised general function of BCSFB in Alzheimer's disease ${ }^{83,84}$, that then b) allows more serum autoantibodies to reach CSFbrain. Therapeutic targeting and stabilization of a) above may attenuate pathophysiology associated with b).

\section{Helicobacter bacterial disruption of choroid plexus}

Gut microbiotica profile shifting is linked to disorders such as Parkinson's disease. This linkage includes CP inflammation in pathophysiologic pathways. Bacterial infection by Helicobacter suis permeabilizes the gastrointestinal tract; in mice, this leads to systemic infection that spreads to CNS causing microglial activation ${ }^{79}$. Helicobacter $s$. inflames CP, opening up the BCSFB gateway for microbe penetration of the CSF-brain nexus ${ }^{21,85}$. Cognitive loss then occurs. (Streptococcus suis also invades human CP epithelium, from the basolateral side $^{86,87}$.) Information is needed for vagal signal contribution ${ }^{88}$, and time points as well as permeability measures for this CNS disturbance.

The Helicobacter $s$. inflammation and dysfunction observed in BCSFB are not found in endothelial $\mathrm{BBB}^{79}$. Helicobacter disablement of BCSFB specifically identifies $\mathrm{CP}$ as a 'causality' in stomach infection disorders giving rise to CSF-brain inflammation that hurts cognition. Thwarting Helicobacter's adverse effects on CP may help stave off cognitive and neurodegenerative problems stemming from gastrointestinal microbiotic disorders.

\section{Choroidal toll-like receptor activity and neuroinflammation}

Central conditions that inappropriately stimulate toll-like receptors devastate neural functions. Infectioninduced inflammation in early life gives rise to maldevelopmental issues such as cerebral palsy ${ }^{89}$. Adjusted 
leukocyte/cytokine migration across BCSFB, controlled by toll-like receptor activity, is a significant aspect of the innate immune response (pathogen pattern recognition). Toll-like receptors (TLR) in CP consist of 9 identified transcripts ${ }^{90}$ for receptor subtypes. TLR2 activity promotes neuroinflammation and neuronal damage ${ }^{91}$. Clinicians need a better understanding of TLR2 impact on BCSFB permeability and leukocyte movement into CSF.

TLR2 stimulation by the synthetic agonist Pam3CSK4 (PC3) increases permeation of monocytes and neutrophils into $\mathrm{CSF}^{92}$. PC3 also augments BCSFB permeability to sucrose. CP inflammation provoked by LPS is substantially less than that by PC3. Low levels of endotoxic LPS in plasma induce cytokine signals in CP that migrate to CSF and brain (Fig. 6). This signaling apprises the CNS about peripheral inflammation. LPS also upregulates TLR2 in CP, and induces pro-inflammatory cytokines ${ }^{93}$. Accordingly, even without pathogen access to CNS, the protective innate immune response is generated in microglia from signals received via $\mathrm{CP}-\mathrm{CSF}$.

Specific activity of TLR2, but not TLR4, promotes neutrophil and monocyte infiltration into the CSF and downstream arachnoid membrane. This is mediated largely by the $\mathrm{BCSFB}^{92}$. Such leukocyte trafficking prompts exploration with synthetic lipopeptides on how inflammatory reactions hurt the developing brain. TLR2 holds special interest in analyzing lipopeptidedriven neurodegeneration ${ }^{91}$. Antagonizing toll receptor activity at BCSFB may limit harm by TLR2-associated neuroinflammation on vulnerable regions.

\section{Choroid plexus-CSF gateway for trypanosomes}

Trypanosome inducers of African sleeping sickness initially and rapidly enter CSF, but not brain, after intraperitoneal injection. Trypanosoma brucei readily passes through the $\mathrm{BCSFB}^{94}$, en route to eventually infecting brain following passage via ventricular CSF flow into SAS (Figs. 2 and 6). This microbe accesses the Virchow-Robin spaces, that are entry loci for subarachnoid CSF flow into cerebral paravascular spaces. (Trypanosomes, activators of lymphocytes, also extensively accumulate in the dura ${ }^{95}$, likely gaining access to dura mater from the blood side.) Trypanosome counts in blood-free CSF are cyclical and proportional to trypanosome infection density of the blood.

There is meagre evidence that trypanosomes pass directly and extensively across BBB. This needs resolution however by newer, sensitive methodologies. To date the best evidence is that trypanosomes penetrate the CNS predominantly by initial CSF access, following which there is convective distribution into brain to set up the second stage of African sleeping sickness. A potential pharmacologic control point for attenuating CNS damage is to block passage of trypanosomes through BCSFB.

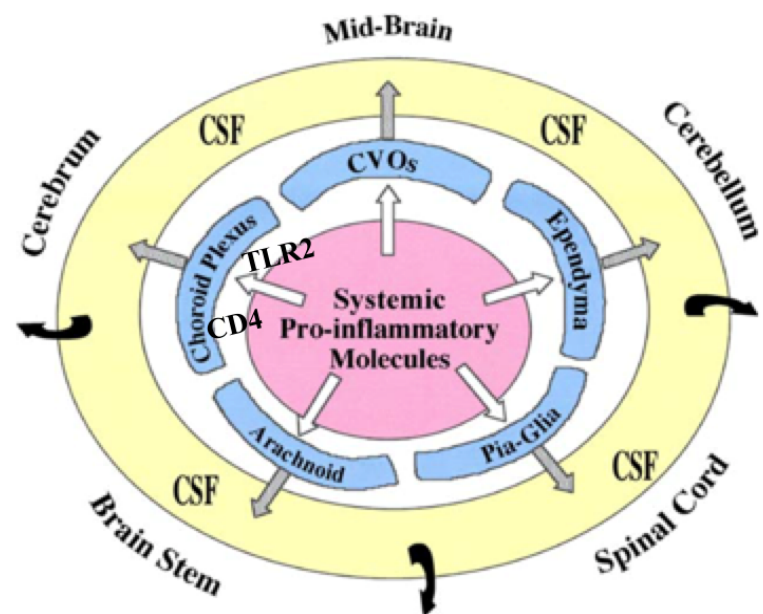

Figure 6: Outward spreading of signaling cytokines into CSF-brain, following serum immune molecule stimulation of CP cytokine receptors TLR2 and CD4. When stimulated by serum immune molecules, e.g., LPS (white arrows), toll like receptors in CP (and other CSF-bordering cells) respond by 'transducing' the peripheral signal into synthesis of epithelial cytokines. Receptors for TL2, TL4 and CD4 expressed in CP and circumventricular organs receive cytokine signals from immune elements in blood perfusing the plexus. Some of the newly-manufactured cytokines in the choroid cell, as part of the innate immune response, are released (gray arrows) into ventricular CSF. Volume transmission conveys cytokine signals in CSF to brain targets (curved black arrows) in various regions. Thus, CNS (e.g., microglia) is apprised of peripheral immune status, without the cytokine, leukocyte or microbe in serum penetrating the BCSFB. Adapted from Johanson et al., Pharm. Res. 2005; 22:1011-37.

\section{Cholesterol and choroidal transport of amyloid}

Knowledge of Alzheimer pathophysiology is more complex $^{96}$ than the original concept of neuronal $A \beta$ production vs. BBB clearance. It is unlikely that any single factor is explanatory. Other complicating factors for neurodegeneration are under consideration both at the systemic (glymphatic drainage) and molecular level (cholesterol). The glymphatic system, involving astrocyte transport, is a "front end" for waste clearance; it is connected downstream to a valid network in the cervical lymphatics ${ }^{97}$. Impaired drainage of $A \beta$ in glymphatic fluid into cervical lymph is likely a contributing factor in Alzheimer pathophysiology, but awaits corroboration ${ }^{98}$.

$\mathrm{A} \beta$ distribution within CNS relates to cholesterol homeostasis and transport at the barriers. Imbalanced cholesterol metabolism/transport in CP-CSF may harm brain. APOE regulates lipid distribution between plasma and body compartments, e.g., CSF and brain. Free cholesterol exchange across BBB and BCSFB favorably affects net $A \beta$ extrusion from $\mathrm{CNS}^{99}$. Individuals hetero- or homozygous for the APOE4 allele have greater vulnerability for disrupted barriers.

In choroidal epithelium, cholesterol release across 
the apical membrane into CSF is less efficient by APOE4 modulation than APOE3 ${ }^{100}$. Less APOE4-dependent extrusion leads to cholesterol accumulation in choroidal epithelium, possibly harming BCSFB functions. $\mathrm{A} \beta$ concentration stability in CNS depends on cholesterol homeostasis. It seems worthwhile to identify $A \beta$ and cholesterol-stabilizing drugs, such as bexarotene (a stimulator of APOE4-mediated BBB cholesterol efflux) ${ }^{99}$, for moderating Alzheimer's disease. Improving CP-CSF BBB cholesterol transport may help to limit $A \beta$-linked neurodegeneration ${ }^{100}$.

\section{Concurrent Actions Associated with $\mathrm{BBB}$ and $\mathrm{BCSFB}$}

How best to assess the permeability of substances distributing across cerebral capillaries into interstitium vs. those through CP into CSF? Uptake of Evans blue-albumin complex by brain shortly after intravenous injection reliably evaluates barrier leakiness. In clinical analysis of dementia, enhanced CSF/SER for albumin is equated to augmented 'BBB permeability'101. Almost certainly, some albumin in brain interstitium (from BBB leakage) diffuses into CSF. Still, steady-state CSF/serum albumin values primarily reflect BCSFB permeability ${ }^{64}$.

Although some investigators lump brain capillaries and CP together generically under the 'BBB' category, this is inappropriate because of the fundamentally different properties of these endothelial vs. epithelial barriers. Accordingly, BBB refers specifically to the cerebral microvessel wall, whereas BCSFB typically designates the CP epithelial membrane.

Discussed below are studies demonstrating altered BCSFB and BBB, occurring to different degrees in the various regional analyses. Time course and severity of barrier modifications impact disease progression as well as remediation.

\section{Helminthic meningitis}

The BCSFB undergoes severe disruption in lungworminduced meningitis. This is contracted by ingesting raw snails with larvae of Angiostrongylus cantonensis. Centrally, a cardinal feature of this disorder is inflamed, impaired CP. CSF has an elevated eosinophil count. Eosinophilic inflammation disrupts BCSFB $^{102}$, increasing CSF protein, albumin, plasminogen activator, matrix metalloproteinases (MMP-9 \& MMP-12), TNF $\alpha$ and claudin-5. Inflammatory molecules and eosinophils flow via ventricular CSF outward to the distal meninges. Serious infection of the arachnoid membrane usually results.

Choroid plexus disruption is central to this helminthic disorder. Eosinophils and cytokines, e.g., TNF $\alpha$, activate molecular pathways at the BCSFB. This leads to NF-kB stimulated matrix metalloproteinase MMP-9. Claudin-5 tight junctions disassemble; enhanced paracellular permeability ensues. Experimental inhibition of NF-kB/ MMP-9 cascading attenuates BCSFB breakdown and reduces CSF protein, leukocyte number, cytokines, and other CP-derived injury molecules ${ }^{103}$. MMP-knockout animals have less perturbed CSF, reduced brain immune cell aggregates, and less virulent meningitis from Angiostrongylus infection ${ }^{104}$. Augmented hepatic growth factor in $\mathrm{CP}^{-\mathrm{CSF}^{105}}$ likely promotes $\mathrm{BCSFB}$ repair in the subsiding infection.

That the BBB is also injured in this meningo-encephalitis disorder is revealed by augmented MRI signals ${ }^{106}$ and Evans blue staining of animal-model brains. Additional investigations, however, should characterize effects of Angiostrongylus $c$. on the cerebrovasculature altered by eosinophilia reactive to larvae. Resveratrol, corticosteroids and anti-helminthic agents reverse CP damage and CSF levels of elevated cytokines ${ }^{102}$. Most CNS knowledge on this meningitis disorder comes from CSF parameters, largely reflecting BCSFB pathophysiology. Pharmacology efforts to control lungworm-associated neuropathology should include drug-manipulated CP.

\section{Ischemia/stroke damage to the barriers}

Reduced CP perfusion exacts a rapid, heavy toll on the BCSFB. Hippocampal CA regions protected by BBB also incur great damage, by a delayed response (at 48 hr). When highly-metabolizing CP is deprived of $\mathrm{O}_{2}$, the BCSFB epithelial syncytium disintegrates. In addition to traumatizing the hippocampal CA regions ${ }^{22}$, transient forebrain ischemia devastates rat $\mathrm{CP}$ epithelial lining ${ }^{20}$. Obliterated microvilli, swollen barrier cells, and formed cytoplasmic vacuoles (from $10 \mathrm{~min}$ of interrupted blood flow and reperfusion) manifest the CP disintegration ${ }^{20}$. Remarkably, the ischemia-injured epithelium recovers by $24 \mathrm{hr}$. This remarkable recovery is aided by trophic/ healing effects of hormones and growth factors secreted by $\mathrm{CP}^{107}$ (Table 2).

A breached BCSFB, reflected by a 3-fold increase in ${ }^{3} \mathrm{H}$-inulin permeability, was noted by Ennis et al. at hour 6 following vessel-occlusion ischemia. They posited that neuronal death in hippocampus at $48 \mathrm{hr}$ was worsened by BCSFB leakiness shortly after stroke/ischemia induction ${ }^{108}$. This notion is supported by the observation that, during ongoing transient forebrain ischemia, apoptosis in rat $\mathrm{CP}$ precedes neuron death in $\mathrm{CA} 1^{109}$. This temporal sequence fits the stroke model of delayed hippocampal injury relating to the earlier-occurring harm of the nearby BCSFB. Still, factors secreted by $\mathrm{CP}$, such as cystatin $\mathrm{C}$ protease inhibitor, likely counter certain hippocampal damage ${ }^{110}$.

How is hippocampus impacted by altered CP transport? Stroke-induced breaching of the BCSFB opens up a 'pathophysiologic portal' through which proteins, cytokines and unneeded plasma molecules freely pass into 
the ventricles. Water osmotically follows molecules leaking into CSF. Ventriculomegaly results; and brain regions proximate to CSF are destabilized. An inflammatory stimulus, co-existing with ischemia/hypoxia, may exacerbate stroke damage by down-regulating the CP Nrf2 anti-oxidant system ${ }^{111}$.

Stroke harm to BCSFB prompts plasma-to-CP-toCSF homeostatic trafficking of monocyte-induced macrophages ${ }^{112}$. When anti-inflammatory M2 macrophages are injected into CSF, the adverse impact of stroke on mouse CNS decreases ${ }^{112}$. M2 trafficking across CP into CSF also heals spinal cord injuries distant from BCSFB ${ }^{113}$. Evidence supports a beneficial CSF flow of M2 cells from ventricular CP secretion to downstream regions of SAS ${ }^{113}$. Regimens to increase M2 cells in CSF appear promising to calm inflammation, even in spinal injuries.

\section{Hyperthermia and disrupted barrier integrity}

Elevated temperature increases BCSFB-BBB permeability ${ }^{18}$, likely by rupturing tight junctions between barrier cells. Whole body hyperthermia $\left(38^{\circ} \mathrm{C}\right)$ for $2 \mathrm{hr}$ in rats opens the BBB to trypan blue, causing edema in hippocampus, hypothalamus, caudate nucleus and thalamus. Simultaneous untoward effects on CP-CSF include choroid cell degeneration, lateral ventriculomegaly and periventricular neuropil destabilization ${ }^{18}$. BBB and BCSFB damage from acute hyperthermia spreads widely in CSF-brain (Fig. 7).

Post-hyperthermia repair includes supportive choroidal growth factors and neurotrophins (Table 2) ${ }^{24}$. CSF flow distributes restorative peptides throughout the ventricles and adjacent brain ${ }^{21}$. Recovery from hyperthermia-induced neuron damage and edema results from ventricular infusion of brain derived neurotrophic factor (BDNF), insulin-like growth factor-1 (IGF-1) and glial derived neurotrophic factor (GDNF) ${ }^{18}$. Hyperthermia effects on CPCSF-brain mimic cortical trauma. Cerebrolysin (a mixture of peptides), upon CSF administration, heals the BCSFB ${ }^{114}$.

Hyperthermia also increases BBB leakiness in other models. An alternating magnetic-field applied to MDCK cells in a transwell enhances paracellular uptake of nanoparticles ${ }^{115}$. Pulsed-wave low-dose ultrasound hyperthermia applied to mice also increases nanodrug uptake by brain; this technique expedites permeation of anti-cancer drugs across $\mathrm{BBB}^{116}$. Elevated temperature likely downregulates claudin proteins. Reduced claudin-1 expression occurs in the in vitro microvascular endothelial cell preparation at $39^{\circ} \mathrm{C}$ (vs. $37^{\circ} \mathrm{C}$ sham control $)^{117}$. Conversely, hypothermia in rats $\left(4 \mathrm{hr}, 33^{\circ} \mathrm{C}\right)$ upregulates tight junction claudin 5; this reduces $\mathrm{BBB}$ damage from endothelium-harming insults ${ }^{118}$. Clearly, pharmacologically-preserved tight junction proteins could well be a clinical strategy to minimize barrier breakdown in hyperthermia and other disorders. Tight junction targeting is also relevant for $\mathrm{CP}^{12,119}$.

\section{Central leptin hormonal resistance and obesity}

Leptin upregulates in response to abdominal fat overload. Secreted by adipocytes, leptin targets the hypothalamus for neuroendocrine signal integration of energy metabolism and thermogenesis. Leptin's role in food intake and weight control commands great interest. The leptin receptor was first cloned for CP.

Leptin accesses the CNS by high-affinity transport at the BBB and BCSFB ${ }^{120,121}$. Endothelial megalin expression facilitates leptin (and insulin) transport into brain to control obesity ${ }^{122}$. A saturable carrier $^{123}$ moves leptin across BCSFB into the ventricles; megalin (LRP-2) binding mediates transport ${ }^{124}$. Leptin flows via CSF streaming to the hypothalamic arcuate nucleus. A second route for leptin to the arcuate is by saturable BBB transport at the capillary interface ${ }^{125}$. Do both routes cover similar cell populations in hypothalamus? The need for two delivery pathways for plasma and CSF leptin to access the arcuate nucleus awaits elucidation.

Leptin's modulation of arcuate neurons helps body weight control by feedback for upregulated adipocyte lipolysis. Resistance to leptin as a weight-regulating hormone is in two forms: i) impaired leptin transport

\begin{tabular}{|c|c|c|c|c|}
\hline $\begin{array}{l}\text { Major } \\
\text { insult to } \\
\text { choroidal } \\
\text { BCSFB }\end{array}$ & $\begin{array}{l}\text { CP edema, } \\
\text { vacuoles \& } \\
\text { TJ rupture }\end{array}$ & $\begin{array}{l}\text { Protein \& } \\
\text { cytokine } \\
\text { leakage } \\
\text { into CSF }\end{array}$ & $\begin{array}{l}\text { Ventricles } \\
\text { take up } \\
\mathrm{H}_{2} \mathrm{O} \text { and } \\
\text { expand }\end{array}$ & $\begin{array}{l}\text { Ependyma, } \\
\text { SVZ are } \\
\text { destabilized; } \\
\text { brain edema }\end{array}$ \\
\hline
\end{tabular}

Figure 7: Pathophysiologic sequelae in CP-CSF-ependyma are similar for forebrain ischemia, hyperthermia and traumatic brain injury. CP becomes edematous as local fluid homeostatic mechanisms fail. Epithelial cells suffer from the insult, vacuoles form, and tight junctions (TJ) break down, leading to enhanced paracellular permeability of the BCSFB. Plasma proteins, including albumin, and cytokines leak into CSF. Augmented CSF oncotic pressure draws water into the ventricles, expanding them. The consequent ventriculomegaly, along with harmful concentration of plasma proteins and pro-inflammatory cytokines, disrupts the ependymal wall. Transependymal penetration of elevated CSF cytokines, proteins and other toxic molecules destabilizes neurogenic niches in subventricular zone (SVZ) and hippocampus. Edema/neurochemical alterations in the periventricular and hippocampal regions, when severe, distort cognitive and behavioral processes. See refs. 18, 19, 20, 22, 24, 107, 108,110 and 114. 
across BBB and/or BCSFB into hypothalamus, and ii) reduced sensitivity of leptin receptors and diminished arcuate signaling. Leptin resistance in pregnancy, manifesting as hyperphagia (increased food intake), results from decreased leptin transport across barriers (transwell analysis ${ }^{126}$ and from altered hypothalamic signaling. The central appetite-enhancing activity in lactation functionally relates to prolactin modulation of arcuate neurons ${ }^{127}$.

On the other hand, food ingestion is spontaneously less in Lou/C rats. Hypophagia in Lou/C is not due to enhanced expression of $\mathrm{CP}$ leptin receptors. Triglyceride changes suggest augmented leptin transport across BBB ${ }^{128}$. Lou/C rats also display improved hypothalamic signaling (increased pSTAT3/STAT3) and less leptin down-regulating cytokine signaling 3 (SOCS3).

Leptin actions intertwine with carbohydrate and fat metabolism. Hyperglycemia likely upregulates barrier leptin transporters, while hypoglycemia downregulates. Chronic early-life stress (mice) increases CP leptin mRNA. Putatively this is central adaptation to early-life stress, with ties to obesity vulnerability persisting into adulthood $^{129}$. Rats with leptin receptor ( $\mathrm{db} / \mathrm{db}$ gene) knockout by CRISPR/Cas9 display an obesity phenotype and carbohydrate distortions. Neutralizing antibodies, e.g., 9F8 against ObR leptin receptor, reveal that ObR may not be the primary leptin transporter in BBB (endothelial monolayer testing $)^{130}$. Ablation by CRISPR/KnockOut and antibody neutralization should elucidate leptin physiology/ pharmacology.

CNS barriers to leptin uptake, i.e., a form of leptin resistance, are overcome by novel pharmacology. Endogenous leptin fails when impaired transporters develop in obesity. The drug leptin has been modified with amphiphilic block copolymers: ethylene and propylene oxides. An example is Pluronic P85 that readily permeates the BBB. Thus the leptin conjugate P85 effectively bypasses leptin transporters and reduces food intake in obesity ${ }^{131}$. Intranasal administration (nose-to-brain) also circumvents CNS barriers by directly accessing CSF. The $\mathrm{N}$ terminal amine of leptin conjugated with $\mathrm{P} 85$ reaches hypothalamus and binds with higher affinity to leptin receptors. Utilizing non-barrier routes and specifically-designed molecular conjugates of leptin hold promise for new tacks for obesityremediating pharmacology ${ }^{132}$.

\section{Glucagon-like peptide improvement of barriers injured by diabetes}

Diabetes mellitus injures the $\mathrm{BBB}$, increasing permeability and impairing cognition. BBB damage plays a significant role in diabetes-dependent $t^{133}$ neurodegeneration, stroke, and especially the combined disorders ${ }^{134}$. Emergence of the 'CNS diabetes' concept begs the question whether insulin-system malfunction alters $C P$ glucose transport, as a possible primary disorder in brain. D-Glucose transport at the BBB supplies neurons with energy substrate whereas glucose uptake at the BCSFB supports choroid epithelial metabolism; thus, evidence is lacking that choroidal D-glucose transport, blood to CSF, impacts neuronal glucose metabolism ${ }^{53}$ in health or Type 3 diabetes/neurodegeneration. Still, diabetes-altered CP ion transporter expression ${ }^{135}$ may affect cerebral functions by modifying CSF homeostasis/dynamics.

Deficient insulin secretion destabilizes the CNS. Glucagon-like peptide-1 (GLP-1), originating in intestinal cells, reduces insulin requirement in diabetes. GLP-1 diffuses rapidly across the $\mathrm{BBB}^{136}$, and is of interest in satiety models (suppressed food intake) and as a neuroprotectant.

Diabetes (streptozotocin) in rats reduces hippocampal expression of BBB tight junction proteins: occludin and claudin 5. Aquaporin 4 levels also decrease. However, treatment with the GLP-1 agonist, exendin, promotes recovery of occludin and aquaporin 4 in hippocampal microvessels. Diabetic rats also suffer losses in CP levels of claudin 2 and aquaporin 1; again, exendin reverses these protein diminutions. Exendin also restores BCSFB and BBB to normal permeability in streptozotocin-induced diabetes $^{137}$.

GLP-1 impacts renal fluid-regulatory mechanisms by lowering Na-K-ATPase activity, and exerting anti-oxidant and anti-inflammatory effects ${ }^{138}$. This prompted Botfield et al. to investigate exendin effects on $\mathrm{CP}$, the so-called 'CNS kidney'. GLP-1 receptors in CP bind exendin, decreasing the Na-K-ATPase activity tied to CSF formation. Predictably, GLP-1 reduced intracranial pressure in hydrocephalic rats $^{139}$. GLP-1 inhibition was prevented with GLP-1 receptor antagonists. GLP-1 is in a new class of agents with remediation potential for elevated intracranial pressure.

\section{LRP-1, tight junctions and amyloid removal: CP-CSF vs. BBB}

A major transporter for clearing $A \beta$ from CNS is LRP1 (low density lipoprotein receptor-related protein 1 ). $A \beta$ removal by LRP- 1 occurs at the CNS-facing aspect of barriers: BBB abluminal and BCSFB apical. Combined, these extrusion systems normally remove $A \beta$ sufficiently to prevent central buildup. LRP-1 also modulates tight junction proteins and remodels ECF matrix ${ }^{140}$.

Tight junctions are vulnerable to pro-inflammatory cytokines. Intestinal-derived microbial metabolites in the circulation alter tight junctions. Propionate protects while LPS (elevated in Alzheimer's) damages tight junctions. Astragaloside protects tight junction injury from LPS ${ }^{141}$, likely by anti-oxidation. Such microbial products and their antagonists (to preserve tight junctions) deserve attention for managing the gut-brain axis in neurodegenerative 
diseases $^{142}$. Regulating barrier permeability (tight junctions) and $A \beta$ transport (by LRP-1) is a dual challenge in managing neurodegeneration.

With aging and progression of late-onset Alzheimer's disease, the $\mathrm{BBB}$ transporters extrude less $A \beta$ into blood $^{143,144}$. Subsequent retention of $A \beta$ by cerebral interstitium predisposes towards $A \beta$ plaque formation and neuroinflammation. LRP- 1 dysfunction in A $\beta$ removal, exacerbated by inflammation, is protected by exogenous anti-oxidant $\mathrm{N}$-acetyl-cysteine ${ }^{145}$. This benefit for LRP-1 may contribute to $\mathrm{N}$-acetyl-cysteine as a neuroprotectant $\mathrm{t}^{146}$.

Novel regimens are sought to enhance LRP-1 transporter strength in neurodegeneration. Vitamin D upregulates LRP-1 in vivo and in vitro. Therefore vitamin $D$ supplementation holds promise for enhancing $A \beta$ clearance to stall Alzheimer's progression ${ }^{147}$. LRP-1, in turn, upregulates claudin expression at barriers. MicroRNA 183 also modifies claudin expression ${ }^{148}$. Therefore, stabilizing LRP-1 and tight junctions by modulators (and antagonists) may prove useful for regulating barrier integrity in the central and peripheral nervous systems ${ }^{148}$.

LRP-1 in CP removes $A \beta^{40}$ from ventricular $\mathrm{CSF}^{149}$, thereby preventing CSF $A \beta$ concentration from rising. Reduced expression of CP LRP-1 from toxicity (aluminum) elevates $A \beta^{42}$ in CSF. Cognitive deficits result ${ }^{150}$. In normal aging ${ }^{151}$ and Alzheimer's ${ }^{152}$, CP LRP-1 expression increases; conversely, BBB LRP-1 expression decreases. Therefore choroidal LRP-1 extrusion of $A \beta$ is a 'backup' in aging and neurodegeneration ${ }^{153}$ when BBB LRP-1 extrusion of $A \beta$ fails. Accordingly, this rescuing feature of BCSFB in aging is a reserve 'transport buffer' to attenuate $A \beta$ buildup. Pharmacologic preservation of $\mathrm{CP} / \mathrm{BBB}$ transporters and tight junctions in late life would almost certainly help to stabilize neuronal function.

\section{Conclusion}

Attention to the CP-CSF's role in neural homeostasis and disease perturbation is steadily rising to the interest level for BBB. Unique physiologic properties of the BCSFB and CSF are continuously being uncovered. Supply to the brain of several endogenous substrates (e.g., vitamin $C$, folate, transthyretin), transported by BCSFB but not BBB, critically depends on a healthy CP. Transporters to maintain CSF oligopeptide concentrations and acid-base balance are part of CP (but not) BBB expression. Again, CSF constancy and thus brain ECF stability depend on bidirectional blood-CSF transport. Further exploration of homeostatic mechanisms expressed at the CP interface is highly desirable to advance neuromedicine.

Mechanistic information for CP extracellular matrix sheds light on leukocyte penetration from microvessels and tight junction regulation. Macrophages and dendritic cells accumulate in the choroidal interstitium, prior to movement into CSF. BCSFB tight junctions are vulnerable to disintegration by matrix metalloproteinase and other injury molecules upregulated in forebrain ischemia, hyperthermia, diabetes and helminthic meningitis. Depleted claudin proteins weaken the BCSFB, permitting paracellular infiltration of macromolecules into CSF. Claudin pharmacology advances will expand the therapeutic armamentarium to prevent BCSFB leakage.

Upon leaking into CSF, the leukocytes and microbial products distribute widely. CSF streams through the ventricles and SAS, where the Virchow-Robin spaces around major penetrating vessels admit CSF-borne materials into perivascular spaces. This long CSF pathway to brain begins in $\mathrm{CP}$, an important therapeutic 'access point' to minimize immunopathologic spreading. CSF distribution is therefore a principal mediator in regulating the 'upstream' CP expeditiously to obtain a more favorable 'downstream' perivascular/glymphatic composition/distribution.

Neuropathologically, CP is both a target and a gateway for throughput of microbes and bacterial products (LPS) into CSF. LPS research on the BBB and BCSFB identifies the structural components of barrier breakdown, allowing the permeating LPS to provoke parenchymal inflammation and neurodegeneration. LPS experimentation, including transcriptomics, is generating new insights on sickness behavior, acute-phase proteins in CP-CSF, and sepsisassociated encephalopathy. Encouraging leads are identifying specific pharmacologic targets (MMP8 protease inhibitors and Nrf2 antioxidant activators) to curtail barrier damage from infective/inflammatory oxidative disorders.

Neuroinflammation induced by microbes threatens neuronal wellbeing. Undue bacterial or cytokine battering of $\mathrm{CP}$ destabilizes the $\mathrm{BCSFB}$, leading to cerebral inflammation. Compromised BCSFB integrity (Helicobacter infection and helminthic meningitis) admits cytokines and leukocytes into CSF-brain. Cytokine spreading by CSF volume transmission activates microglia, promoting 'sickness behavior'. A worthwhile goal is to counter low grade and virulent infections (SAE) in CNS by manipulating the BCSFB with immunopharmacology. For healing widespread neural dysfunction, it seems feasible to treat primary $\mathrm{CP}$ injuries resulting from microbes (trypanosomes) and autoimmune antibodies (NPSLE and NMDA). Accordingly, BCSFB pharmacotherapy has great potential for controlling neuroinflammation.

A range of disorders compromises $\mathrm{BCSFB}$ and $\mathrm{BBB}$, but to variable degrees. Forebrain ischemia/stroke devastates hippocampal BBB and choroidal epithelium. Focal ischemia in areas remote from CSF damages the local neurovascular networks, without seriously impairing BCSFB. In other pathology, BBB incurs greater damage than CP. Advanced Alzheimer's disease (AD) is characterized by cerebral 
microvessel damage (e.g., downregulated LRP-1 and A $\beta$ removal) but the counterpart LRP-1 expression in CP is expeditiously sustained. Elucidating the persistent LRP-1 expression in the aging/AD BCSFB may open a new vista to combat neurodegeneration.

New basic knowledge prompts fresh pharmacologic approaches to counter brain deficits. CP's strategic position (CNS interior; Fig. 8) assures distributional access to many regions contacting CSF. The unique choroid epithelial transporter repertoire facilitates movement of many organic molecules into CSF. An exciting prospect is bile acid use for neurological diseases. Taurosodeoxycholic acid stimulates Nrf2 expression. This stabilizes BCSFB against excessive oxidation/apoptosis, while concurrently suppressing Parkinson's disease development. Further research will reveal if bile acid penetration into CSF is passive, or active via OATP 154,155 ? Development of bile acid analogs will hopefully be useful in thwarting Parkinson's pathology.

Translational neuromedicine now includes exploiting BCSFB transport mechanisms; and using direct CSF access,

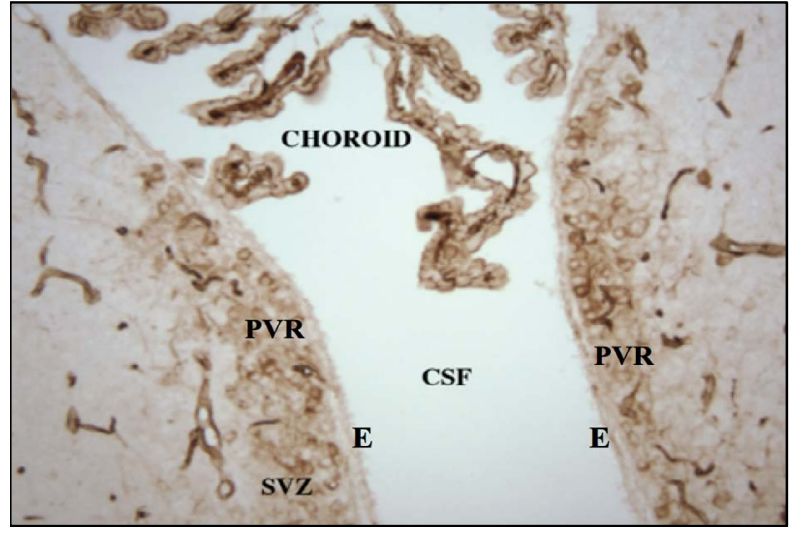

Figure 8: Choroidal villi fan out to a dynamically-changing 'net' with vast surface area for secretion/reabsorption throughout ventricular fluid. CP tissues are positioned near periventricular regions (PVR) that mediate neural, axonal, neurogenic and immunologic functions. CSF-borne substances distribute readily across ependyma (E) into periventricular regions (PVR). CP-CSF furnishes brain interior with many growth factors/ neurotrophins to: i) expedite neurogenesis/fetal brain growth, ii) foster maintenance of neurons/glia in health, and iii) help brain adjust to disorders of aging/senescence. In disabled conditions, upregulated secretions by CP heal injured neuronal networks. Opportunities exist for new pharmacologic and transgenetic manipulations of BCSFB to furnish restorative agents to regions around ventricular CSF. Such areas contain networks mediating cognitive, endocrine, motor, and pain processes. Drugs designed to access CSF by way of CP also potentially modulate 'downstream' cortical (paravascular inflow to brain interior) and spinal cord sites. Image is of adult rat lateral ventricle $\mathrm{CP}$. intranasally, to deliver polypeptides into $\mathrm{CNS}^{156}$. CSF is an extensive circulatory conduit with high potential for drug delivery to neurons/glia. Some challenges, e.g., overcoming leptin resistance in obesity, might be better resolved by targeting CP-CSF as well as BBB. Intranasal presentation of polypeptides (e.g., insulin for AD) for access to the CSFbrain nexus successfully circumvents the BBB. Addressing CNS pathophysiology with CSF neuropharmacology strategies affords novel opportunities for resolving brain disorders.

Identifying specific neuropathology and regional barrier breakdowns is critical for devising neural repair regimens. Dual damage to BBB and CP may require bimodal remediation. Future pharmacology employing designer agents can target specific elements of the neurovascular unit and CP stroma/epithelium. Medicinals manufactured to reconstitute BBB breaches and shore up CP capabilities will likely improve CSF dynamics and benefit cerebral metabolism. Vulnerable patients can benefit from dietary/ medical regimens to strengthen $\mathrm{CP}$ homeostatic reserve (e.g., healthy oxidative balance) in anticipation of highrisk complications. This could have salutary effects on attenuating stroke/seizure episodes. There is room for optimism that finely-controlled barrier transport and permeability will ameliorate many neurologic disorders ${ }^{157}$.

\section{Conflict of Interest Statement}

The authors have no conflicts of interest to declare.

\section{Acknowledgements}

We are grateful to Dr. S. R. Snodgrass for critical reading of the manuscript and helpful comments. Dr. E. Stopa provided neuropathological comments on the Alzheimer's disease information, procured in part from grant support to CEJ (P.I., NIH R01 AG027910). Acknowledgment is kindly due to Dr. P. McMillan for excellent microscopy.

\section{References}

1. Daneman R, Zhou L, Agalliu D, et al. The mouse blood-brain barrier transcriptome: a new resource for understanding the development and function of brain endothelial cells. PloS One. 2010; 5(10): e13741.

2. Bergen AA, Kaing S, ten Brink JB, et al. Gene expression and functional annotation of human choroid plexus epithelium failure in Alzheimer's disease. BMC Genomics. 2015; 16: 956.

3. Janssen SF, van der Spek SJ, Ten Brink JB, et al. Gene expression and functional annotation of the human and mouse choroid plexus epithelium. PloS One. 2013; 8(12): e83345.

4. Zhao F, Ding XY, Wu F, et al. Effects of passage and cryopreservation on neurotrophic factor secretion from choroid plexus epithelial cells. Biomed Rep. 2018; 8(6): 535-9.

5. Huang SL, Wang J, He XJ, et al. Secretion of BDNF and GDNF from free and encapsulated choroid plexus epithelial cells. Neuroscience Letters. 2014; 566: 42-5.

6. Plog BA, Nedergaard M. The glymphatic system in central nervous system health and disease: Past, present, and future. Annu Rev Pathol. 2018; 13: 379-94. 
7. Johanson CE, Duncan JA, 3rd, Klinge PM, et al. Multiplicity of cerebrospinal fluid functions: New challenges in health and disease. Cerebrospinal Fluid Res. 2008; 5: 10.

8. Damkier HH, Brown PD, Praetorius J. Cerebrospinal fluid secretion by the choroid plexus. Physiological Reviews. 2013; 93(4): 1847-92.

9. Brown PD, Davies SL, Speake T, et al. Molecular mechanisms of cerebrospinal fluid production. Neuroscience. 2004; 129(4): 957-70.

10. Mokgokong R, Wang S, Taylor CJ, et al. Ion transporters in brain endothelial cells that contribute to formation of brain interstitial fluid. Pflugers Arch. 2014; 466(5): 887-901.

11. Davson H, Segal M. Physiology of the CSF and Blood-Brain Barriers. Boca Raton, FL: CRC; 1996. p. 822.

12. Johanson CE, Stopa EG, Daiello L, et al. Disrupted blood-CSF barrier to urea and creatinine in mild cognitive impairment and Alzheimer's disease. Journal of Alzheimer's Disease and Parkinsonism. 2018;8:2.

13. Spector R, Johanson CE. Choroid plexus failure in the Kearns-Sayre syndrome. Cerebrospinal Fluid Res. 2010; 7(1): 14.

14. Martins IJ. Autoimmune disease and mitochondrial dysfunction in chronic diseases. Res Chron Dis. 2017; 1(1): 10-2.

15. Wu Q, Gao C, Wang H, et al. Mdivi-1 alleviates blood-brain barrier disruption and cell death in experimental traumatic brain injury by mitigating autophagy dysfunction and mitophagy activation. Int J Biochem Cell Biol.. 2018; 94: 44-55.

16. Batllori M, Molero-Luis M, Ormazabal A, et al. Cerebrospinal fluid monoamines, pterins, and folate in patients with mitochondrial diseases: systematic review and hospital experience. J Inherit Metab Dis. 2018; Jul 4.

17. Liu C, Wang $\mathrm{R}$, Yi $\mathrm{Y}$, et al. Lycopene mitigates $\beta$-amyloid induced inflammatory response and inhibits NF- $\kappa B$ signaling at the choroid plexus in early stages of Alzheimer's disease rats. J Nutr Biochem. 2018; 53: 66-71.

18. Sharma HS, Johanson CE. Intracerebroventricularly administered neurotrophins attenuate blood cerebrospinal fluid barrier breakdown and brain pathology following whole-body hyperthermia: An experimental study in the rat using biochemical and morphological approaches. Annals of the New York Academy of Sciences. 2007; 1122: 112-29.

19. Sharma HS, Duncan JA, Johanson CE. Whole-body hyperthermia in the rat disrupts the blood-cerebrospinal fluid barrier and induces brain edema. Acta Neurochir Suppl. 2006; 96: 426-31.

20. Palm D, Knuckey N, Guglielmo M, et al. Choroid plexus electrolytes and ultrastructure following transient forebrain ischemia. The American Journal of Physiology. 1995; 269(1 Pt 2): R73-9.

21. Johanson CE, Stopa E, McMillan PN, et al. The distributional nexus of choroid plexus to CSF, ependyma and brain: Toxicologic/pathologic phenomena, periventricular destabilization and lesion spread. Toxicologic Pathology. 2011; 39(1): 186-212.

22. Knuckey NW, Finch P, Palm DE, et al. Differential neuronal and astrocytic expression of transforming growth factor beta isoforms in rat hippocampus following transient forebrain ischemia. Brain Res Mol Brain Res. 1996; 40(1): 1-14

23. Walter HJ, Berry M, Hill DJ, et al. Distinct sites of insulin-like growth factor (IGF)-II expression and localization in lesioned rat brain: possible roles of IGF binding proteins (IGFBPs) in the mediation of IGF-II activity. Endocrinology. 1999; 140(1): 520-32.

24. Johanson C, Stopa E, Baird A, et al. Traumatic brain injury and recovery mechanisms: peptide modulation of periventricular neurogenic regions by the choroid plexus-CSF nexus. J Neural Transm (Vienna). 2011; 118(1): 115-33.

25. Johanson CE. Choroid plexus-cerebrospinal fluid transport dynamics: Support of brain health and a role in neurotherapeutics In Translational Neuroscience. Conn PM, ed.: Elsevier Inc., Academic Press; 2017;233-61.

26. Schoknecht K, Shalev H. Blood-brain barrier dysfunction in brain diseases: clinical experience. Epilepsia. 2012; 53 Suppl 6: 7-13.

27. Martins IJ. Antimicrobial activity inactivation and toxic immune reactions induce epilepsy in humans. Journal of Medical Discovery. 2017; $2: 1-7$

28. Newton S, Collier E, Hunsberger J, et al. Gene profile of electroconvulsive seizures: induction of neurotrophic and angiogenic factors. J Neurosci. 2003; 23(34):10841-51.

29. Girgenti M, Hunsberger J, Duman C, et al. Erythropoietin induction by electroconvulsive seizure, gene regulation, and antidepressant-like behavioral effects. Biol Psychiatry. 2009; 66(3): 267-74.

30. Varatharaj A, Galea I. The blood-brain barrier in systemic inflammation. Brain Behav Immun. 2017; 60: 1-12.

31. Liu W, Wang Z, Wang Y, et al. Increasing the permeability of the bloodbrain barrier in three different models in vivo. CNS Neurosci Ther 2015 ; $21(7)$ : 568-74

32. Banks W, Gray A, Erickson M, et al. Lipopolysaccharide-induced blood-brain barrier disruption: roles of cyclooxygenase, oxidative stress, neuroinflammation, and elements of the neurovascular unit. J Neuroinflammation. 2015; 12: 223.

33. Chastre A, Bélanger M, Nguyen B, et al. Lipopolysaccharide precipitates hepatic encephalopathy and increases blood-brain barrier permeability in mice with acute liver failure Liver Int. 2014; 34(3): 353-61.

34. Jangula A, Murphy E. Lipopolysaccharide-induced blood brain barrier permeability is enhanced by alpha-synuclein expression. Neurosci Lett. 2013; 551: 23-7.

35. Kowalewska M, Herman A, Szczepkowska A, et al. The effect of melatonin from slow-release implants on basic and TLR-4-mediated gene expression of inflammatory cytokines and their receptors in the choroid plexus in ewes. Res Vet Sci. 2017; 113: 50-5

36. Marques F, Sousa JC, Coppola G, et al. Kinetic profile of the transcriptome changes induced in the choroid plexus by peripheral inflammation. J Cereb Blood Flow Metab. 2009; 29(5): 921-32.

37. Di Filippo PA, Saulo T. Lannes ST, et al. Acute phase proteins in serum and cerebrospinal fluid in healthy cattle: possible use for assessment of neurological diseases. Pesq Vet Bras. 2018; 38(4): 779-84.

38. Hasegawa-Ishii S, Inaba M, Umegaki H, et al. Endotoxemia-induced cytokine-mediated responses of hippocampal astrocytes transmitted by cells of the brain-immune interface. Sci Rep. 2016; 6: 25457

39. Mazeraud A, Pascal Q, Verdonk F, et al. Neuroanatomy and physiology of brain dysfunction in sepsis. Clin Chest Med. 2016; 37(2): 333-45.

40. Ji M, Tang H, Luo D, et al. Environmental conditions differentially affect neurobehavioral outcomes in a mouse model of sepsis-associated encephalopathy. Oncotarget. 2017; 8(47):82376-89.

41. Vandenbroucke R, Dejonckheere E, Van Lint $P$, et al. Matrix metalloprotease 8-dependent extracellular matrix cleavage at the blood-CSF barrier contributes to lethality during systemic inflammatory diseases. J Neurosci. 2012; 32 (29):9805-16.

42. Steeland S, Van Ryckeghem S, Vandewalle J, et al. Simultaneous inhibition of tumor necrosis factor receptor 1 and matrix metalloproteinase 8 completely protects against acute inflammation and sepsis. Crit Care Med. 2018; 46 (1): e67-e75.

43. Balusu S, Van Wonterghem E, De Rycke R, et al. Identification of a novel mechanism of blood-brain communication during peripheral inflammation via choroid plexus-derived extracellular vesicles. EMBO Mol Med. 2016; 8(10): 1162-83. 
44. Grapp M, Wrede A, Schweizer M, et al. Choroid plexus transcytosis and exosome shuttling deliver folate into brain parenchyma. Nat Commun. 2013; 4: 2123 .

45. Balusu S, Van Wonterghem E, De Rycke R, et al. Identification of a novel mechanism of blood-brain communication during peripheral inflammation via choroid plexus-derived extracellular vesicles. EMBO Mol Med. 2016; 8(10): 1162-83.

46. Ha D, Yang N, Nadithe V. Exosomes as therapeutic drug carriers and delivery vehicles across biological membranes: current perspectives and future challenges. Acta Pharm Sin B. 2016; 6(4): 287-96.

47. Johanson $C$, Johanson N. Merging transport data for choroid plexus with blood-brain barrier to model CNS homeostasis and disease more effectively. CNS Neurol Disord Drug Targets. 2016; 15(9): 1151-80.

48. Chen X, Loryan I, Payan M, et al. Effect of transporter inhibition on the distribution of cefadroxil in rat brain Fluids Barriers CNS.. 2014; $11(1): 25$

49. Shen H, Smith D, Keep R, et al. Targeted disruption of the PEPT2 gene markedly reduces dipeptide uptake in choroid plexus. J Biol Chem. 2003; 278 (7): 4786-91.

50. Carvey P, Hendey B, Monahan A. The blood-brain barrier in neurodegenerative disease: a rhetorical perspective. J Neurochem. 2009; 111(2): 291-314.

51. Rodriguez EM, Blazquez JL, Guerra M. The design of barriers in the hypothalamus allows the median eminence and the arcuate nucleus to enjoy private milieus: the former opens to the portal blood and the latter to the cerebrospinal fluid. Peptides. 2010; 31(4): 757-76.

52. Spector R, Johanson CE. The nexus of vitamin homeostasis and DNA synthesis and modification in mammalian brain. Mol Brain. 2014; 7: 3

53. Keep R, Smith D. Choroid plexus transport: gene deletion studies. Fluids Barriers CNS. 2011; 8(1): 26.

54. Cho PY, Joshi G, Johnson JA, et al. Transthyretin-derived peptides as $\beta$-amyloid inhibitors. ACS Chem Neurosci. 2014; 5(7): 542-51.

55. Johanson CE. Fluid-forming functions of the choroid plexus: What is the role of aquaporin-1? In The Blood-Brain Barrier in Health and Disease, Vol. 1: Morphology, Biology and Immune Function. K. Dorovini-Zis, ed., CRC Press, 2015; pp. 140-71.

56. Johanson CE, Woodbury DM. Uptake of [14C]urea by the in vivo choroid plexus--cerebrospinal fluid--brain system: Identification of sites of molecular sieving. The Journal of Physiology. 1978; 275: 167 76.

57. Engelhardt B, Ransohoff RM. Capture, crawl, cross: the T cell code to breach the blood-brain barriers. Trends Immunol. 2012; 33(12): 57989.

58. Erickson MA, Banks WA. Neuroimmune axes of the blood-brain barriers and blood-brain interfaces: Bases for physiological regulation, disease states, and pharmacological interventions. Pharmacol Rev. 2018; 70(2): 278-314

59. Levine S. Choroid plexus: target for systemic disease and pathway to the brain. Laboratory Investigation; A Journal of Technical Methods and Pathology. 1987; 56(3): 231-3.

60. Dahm T, Adams O, Boettcher S, et al. Strain-dependent effects of clinical echovirus 30 outbreak isolates at the blood-CSF barrier. J Neuroinflammation. 2018; 15(1): 50.

61. Burkala EJ, He J, West JT, et al. Compartmentalization of HIV-1 in the central nervous system: Role of the choroid plexus. AIDS (London, England). 2005; 19(7): 675-84.

62. Chen RL, Kassem NA, Redzic ZB, et al. Age-related changes in choroid plexus and blood-cerebrospinal fluid barrier function in the sheep. Exp Gerontol. 2009; 44(4): 289-96.
63. Johanson CE, Stopa E, McMillan PN. The blood-cerebrospinal fluid barrier: Structure and functional significance. Methods Mol Biol. 2011;686:101-31.

64. Johanson CE, Stopa EG, Daiello L, et al. Disrupted blood-CSF barrier to urea and creatinine in mild cognitive impairment and Alzheimer's disease. J Alzheimer's Disease and Parkinsonism. 8:2.

65. Chen RL. Is it appropriate to use albumin CSF/plasma ratio to assess blood brain barrier permeability. Neurobiology of Aging. 2011; 32(7): 1338-9.

66. Chodobski A, Szmydynger-Chodobska J. Choroid plexus: target for polypeptides and site of their synthesis. Microscopy research and technique. 2001; 52(1): 65-82.

67. Rosenberg GA. Neurological diseases in relation to the blood-brain barrier. J Cereb Blood Flow Metab. 2012; 32(7): 1139-51.

68. Spector R, Keep RF, Snodgrass SR, et al. A balanced view of choroid plexus structure and function: Focus on adult humans. Experimental Neurology. 2015; 267: 78-86

69. Kratzer I, Strazielle N, Saudrais E, et al. Glutathione conjugation at the blood-CSF barrier efficiently prevents exposure of the developing brain fluid environment to blood-borne reactive electrophilic substances. J Neurosci. 2018; 38(14): 3466-79.

70. Damkier HH, Christensen HL, Christensen IB, et al. The murine choroid plexus epithelium expresses the $2 \mathrm{Cl}(-) / \mathrm{H}(+)$ exchanger $\mathrm{ClC}-7$ and $\mathrm{Na}(+) / \mathrm{H}(+)$ exchanger NHE6 in the luminal membrane domain. Am. J Physiol. 2018; 314(4): C439-C48.

71. Tomás J, Santos C, Quintela T, et al. “Tasting” the cerebrospinal fluid Another function of the choroid plexus? Neuroscience. 2016; 320 160-71.

72. De Bock M, Vandenbroucke RE, Decrock E, Culot M, Cecchelli R, Leybaert L. A new angle on blood-CNS interfaces: A role for connexins? FEBS Lett. 2014;588(8):1259-70.

73. Quintela T, Albuquerque T, Lundkvist G, et al. The choroid plexus harbors a circadian oscillator modulated by estrogens. Chronobiol Int. 2018; 35(2): 270-9.

74. Myung J, Schmal C, Hong S, et al. The choroid plexus is an important circadian clock component. Nat Commun. 2018; 9(1): 1062.

75. McMillin M, DeMorrow S. Effects of bile acids on neurological function and disease. FASEB J. 2016; 30(11): 3658-68.

76. Moreira S, Fonseca I, Nunes MJ, et al. Nrf2 activation by tauroursodeoxycholic acid in experimental models of Parkinson's disease. Exp Neurol. 2017;295: 77-87.

77. Xiang J, Alesi GN, Zhou N, et al. Protective effects of isothiocyanates on blood-CSF barrier disruption induced by oxidative stress. . Am J Physiol Regul Integr Comp Physiol. 2012; 303(1): R1-R7.

78. Ahuja M, Ammal Kaidery N, Yang L, et al. Distinct Nrf2 signaling mechanisms of fumaric acid esters and their role in neuroprotection against 1-Methyl-4-Phenyl-1,2,3,6-Tetrahydropyridine-induced experimental Parkinson's-like disease. J Neurosci. 2016; 36(23): 6332-51.

79. Gorlé N, Blaecher C, Bauwens E, et al. The choroid plexus epithelium as a novel player in the stomach-brain axis during Helicobacter infection. Brain Behav Immun. 2018; 69: 35-47.

80. Gelb S, Stock AD, Anzi S, et al. Mechanisms of neuropsychiatric lupus: The relative roles of the blood-cerebrospinal fluid barrier versus blood-brain barrier. J Autoimmun. 2018;91:34-44.

81. Hammer C, Stepniak B, Schneider A, et al. Neuropsychiatric disease relevance of circulating anti-NMDA receptor autoantibodies depends on blood-brain barrier integrity. Molecular Psychiatry. 2014; 19(10): $1143-9$ 
82. Busse M, Kunschmann R, Dobrowolny $H$, et al. Dysfunction of the blood-cerebrospinal fluid-barrier and N-methyl-D-aspartate glutamate receptor antibodies in dementias. Eur Arch Psychiatry Clin Neurosci. 2018; 268(5):483-92.

83. Johanson C, McMillan P, Tavares R, et al. Homeostatic capabilities of the choroid plexus epithelium in Alzheimer's disease. Cerebrospinal Fluid Res. 2004; 1(1): 3

84. Serot JM, Bene MC, Faure GC. Choroid plexus, aging of the brain, and Alzheimer's disease. Front Biosci. 2003; 8: s515-21.

85. Levine S, Sowinski R, Nochlin D. Periventricular localization of a toxic encephalopathy induced by a mechanism involving choroid plexus. Brain Res. 1982; 242(2): 219-25.

86. Schwerk C, Tenenbaum T, Kim KS, et al. The choroid plexus- a multirole player during infectious diseases of the CNS. Front Cell Neurosci. 2015; 9: 80 .

87. Schwerk C, Papandreou T, Schuhmann D, et al. Polar invasion and translocation of Neisseria meningitidis and Streptococcus suis in a novel human model of the blood-cerebrospinal fluid barrier. PloS One. 2012; 7(1): e30069.

88. Carabotti M, Scirocco A, Maselli MA, et al. The gut-brain axis: interactions between enteric microbiota, central and enteric nervous systems. Ann Gastroenterol. 2015; 28(2): 203-9.

89. Strunk T, Inder T, Wang X, et al. Infection-induced inflammation and cerebral injury in preterm infants. Lancet Infect Dis. 2014; 14(8): 751 62

90. Skipor J, Szczepkowska A, Kowalewska M, et al. Profile of toll-like receptor mRNA expression in the choroid plexus in adult ewes. Acta Vet Hung. 2015; 63(1): 69-78.

91. Hoffmann O, Braun JS, Becker D, et al. TLR2 mediates neuroinflammation and neuronal damage. J Immunol. 2007; 178(10): 6476-81.

92. Mottahedin A, Smith PL, Hagberg H, et al. TLR2-mediated leukocyte trafficking to the developing brain. J Leukoc Biol. 2017; 101(1): 297305 .

93. Rivest $\mathrm{S}$. Molecular insights on the cerebral innate immune system. Brain Behav Immun. 2003; 17(1): 13-9.

94. Mogk S, Boßelmann CM, Mudogo CN, et al. African trypanosomes and brain infection - the unsolved question. Biol Rev Camb Philos Soc 2017; 92(3): 1675-87

95. Coles JA, Myburgh E, Ritchie R, et al. Intravital imaging of a massive lymphocyte response in the cortical dura of mice after peripheral infection by trypanosomes. PLoS Negl Trop Dis. 2015; 9(4): e0003714.

96. Franceschi C, Garagnani P, Morsiani C, et al. The continuum of aging and age-related diseases: Common mechanisms but different rates. Front Med (Lausanne). 2018; 5: 61.

97. Benveniste H, Liu X, Koundal S, et al. The glymphatic system and waste clearance with brain aging: A review. Gerontology. 2018; Jul 11: 1-14.

98. Smith AJ, Verkman AS. The "glymphatic" mechanism for solute clearance in Alzheimer's disease: game changer or unproven speculation. FASEB J. 2018; 32(2): 543-51.

99. Kuntz M, Candela P, Saint-Pol J, et al. Bexarotene promotes cholesterol efflux and restricts apical-to-basolateral transport of amyloid- $\beta$ peptides in an in vitro model of the human blood-brain barrier. J Alzheimers Dis. 2015; 48(3): 849-62.

100. Fujiyoshi M, Ohtsuki S, Hori S, et al. 24S-hydroxycholesterol induces cholesterol release from choroid plexus epithelial cells in an apical- and apoE isoform-dependent manner concomitantly with the induction of ABCA1 and ABCG1 expression. Journal of Neurochemistry. 2007; 100(4): 968-78.
101. Skillbäck T, Delsing L, Synnergren J, et al. CSF/serum albumin ratio in dementias: a cross-sectional study on 1861 patients. Neurobiology of Aging. 2017; 59: 1-9.

102. Chen AC, Shyu LY, Hsin YL, et al. Resveratrol relieves Angiostrongylus cantonensis - induced meningoencephalitis by activating sirtuin-1. Acta Trop. 2017; 173: 76-84.

103. Chiu PS, Lai SC. Matrix metalloproteinase-9 leads to claudin-5 degradation via the NF- $\kappa$ B pathway in BALB/c mice with eosinophilic meningoencephalitis caused by Angiostrongylus cantonensis. PloS One. 2013; 8(3): e53370.

104. Chiu PS, Lai SC. Matrix metalloproteinase-9 leads to blood-brain barrier leakage in mice with eosinophilic meningoencephalitis caused by Angiostrongylus cantonensis. Acta Trop. 2014; 140: 14150 .

105. Tsai HC, Huang YL, Liu YC, et al. Dynamic changes of hepatocyte growth factor in eosinophilic meningitis caused by Angiostrongylus cantonensis infection. Am J Trop Med Hyg. 2009; 80(6): 980-2.

106. Tsai HC, Chen YS, Yen CM. Human parasitic meningitis caused by Angiostrongylus cantonensis infection in Taiwan. Hawaii J Med Public Health. 2013; 72(6 Suppl 2): 26-7.

107. Johanson CE, Palm DE, Primiano MJ, et al. Choroid plexus recovery after transient forebrain ischemia: role of growth factors and other repair mechanisms. Cellular and Molecular Neurobiology. 2000 20(2): 197-216

108. Ennis SR, Keep RF. The effects of cerebral ischemia on the rat choroid plexus. J Cereb Blood Flow Metab. 2006; 26(5): 675-83.

109. Ferrand-Drake M. Cell death in the choroid plexus following transient forebrain global ischemia in the rat. Microscopy Research and Technique. 2001; 52(1): 130-6.

110. Palm DE, Knuckey NW, Primiano MJ, et al. Cystatin C, a protease inhibitor, in degenerating rat hippocampal neurons following transient forebrain ischemia. Brain Res. 1995; 691(1-2): 1-8.

111. D’Angelo B, Ek CJ, Sandberg M, et al. Expression of the Nrf2-system at the blood-CSF barrier is modulated by neonatal inflammation and hypoxia-ischemia. J Inherit Metab Dis. 2013; 36(3): 479-90.

112. Ge R, Tornero D, Hirota M, et al. Choroid plexus-cerebrospinal fluid route for monocyte-derived macrophages after stroke. J Neuroinflammation. 2017; 14(1): 153.

113. Shechter R, Miller O, Yovel G, et al. Recruitment of beneficial M2 macrophages to injured spinal cord is orchestrated by remote brain choroid plexus. Immunity. 2013; 38(3): 555-69.

114. Sharma HS, Zimmermann-Meinzingen S, Johanson CE. Cerebrolysin reduces blood-cerebrospinal fluid barrier permeability change, brain pathology, and functional deficits following traumatic brain injury in the rat. Annals New York Academy of Sciences. 2010; 1199: 125-37.

115. Dan M, Bae Y, Pittman TA, et al. Alternating magnetic field-induced hyperthermia increases iron oxide nanoparticle cell association/ uptake and flux in blood-brain barrier models. Pharmaceutical Research. 2015; 32(5): 1615-25.

116. Wu SK, Chiang CF, Hsu YH, et al. Pulsed-wave low-dose ultrasound hyperthermia selectively enhances nanodrug delivery and improves antitumor efficacy for brain metastasis of breast cancer. Ultrason Sonochem. 2017; 36: 198-205.

117. Ji C, Wang L, Dai R, et al. Hyperthermia exacerbates the effects of cathepsin L on claudin-1 in a blood-brain barrier model in vitro. Brain Res. 2016; 1631: 72-9.

118. Sun H, Tang Y, Guan X, et al. Effects of selective hypothermia on bloodbrain barrier integrity and tight junction protein expression levels after intracerebral hemorrhage in rats. Biol Chem. 2013; 394(10): 1317-24. 
119. Steeland S, Gorlé N, Vandendriessche C, et al. Counteracting the effects of TNF receptor-1 has therapeutic potential in Alzheimer's disease. EMBO Mol Med. 2018;10(4).

120. Kurrimbux D, Gaffen Z, Farrell CL, et al. The involvement of the bloodbrain and the blood-cerebrospinal fluid barriers in the distribution of leptin into and out of the rat brain. Neuroscience. 2004; 123(2) 527-36.

121. Zlokovic BV, Jovanovic S, Miao W, et al. Differential regulation of leptin transport by the choroid plexus and blood-brain barrier and high affinity transport systems for entry into hypothalamus and across the blood-cerebrospinal fluid barrier. Endocrinology. 2000; 141(4): 1434-41

122. Bartolome F, Antequera D, Tavares E, et al. Obesity and neuroinflammatory phenotype in mice lacking endothelial megalin. J Neuroinflammation. 2017; 14(1): 26.

123. Thomas SA, Preston JE, Wilson MR, et al. Leptin transport at the blood--cerebrospinal fluid barrier using the perfused sheep choroid plexus model. Brain Res. 2001; 895(1-2): 283-90.

124. Dietrich MO, Spuch C, Antequera D, et al. Megalin mediates the transport of leptin across the blood-CSF barrier. Neurobiology of Aging. 2008;29(6):902-12.

125. Banks WA, Kastin AJ, Huang W, et al. Leptin enters the brain by a saturable system independent of insulin. Peptides. 1996; 17(2): 305-11.

126. Trujillo ML, Spuch C, Carro E, et al. Hyperphagia and central mechanisms for leptin resistance during pregnancy. Endocrinology. 2011; 152(4): 1355-65.

127. Szczesna M, Zieba DA. Phenomenon of leptin resistance in seasonal animals: the failure of leptin action in the brain. Domest Anim Endocrinol. 2015; 52: 60-70.

128. Veyrat-Durebex C, Poher AL, Caillon A, et al. Improved leptin sensitivity as a potential candidate responsible for the spontaneous food restriction of the Lou/C rat. PloS One. 2013; 8(9): e73452.

129. Yam KY, Naninck EFG, Abbink MR, et al. Exposure to chronic earlylife stress lastingly alters the adipose tissue, the leptin system and changes the vulnerability to western-style diet later in life in mice. Psychoneuroendocrinology. 2017; 77: 186-95.

130. Gonzalez-Carter D, Goode AE, Fiammengo R, et al. Inhibition of leptin-ObR interaction does not prevent leptin translocation across a human blood-brain barrier model. J Neuroendocrinol. 2016; 28(6).

131. Yi X, Yuan D, Farr SA, et al. Pluronic modified leptin with increased systemic circulation, brain uptake and efficacy for treatment of obesity. J Control Release. 2014; 191: 34-46.

132. Yuan D, Yi X, Zhao Y, et al. Intranasal delivery of N-terminal modified leptin-pluronic conjugate for treatment of obesity. J Control Release. 2017: 263: 172-84

133. Prasad S, Sajja RK, Pooja N, et al. Diabetes mellitus and blood-brain barrier dysfunction: An overview. Journal of Pharmacovigilance. $2014 ; 2: 2$

134. Venkat P, Chopp M, Chen J. Blood-brain barrier disruption, vascular impairment, and ischemia/reperfusion damage in diabetic stroke. J Am Heart Assoc. 2017; 6(6).

135. Egleton RD, Campos CC, Huber JD, et al. Differential effects of diabetes on rat choroid plexus ion transporter expression. Diabetes. 2003 52(6): 1496-501.

136. Kastin AJ, Akerstrom V, Pan W. Interactions of glucagon-like peptide-1 (GLP-1) with the blood-brain barrier. J Mol Neurosci. 2002; 18(1-2): 7-14.

137. Zanotto C, Simão F, Gasparin MS, et al. Exendin-4 reverses biochemical and functional alterations in the blood-brain and blood-CSF barriers in diabetic rats. Mol Neurobiol. 2017; 54(3): 2154-66.
138. Sancar-Bas S, Gezginci-Oktayoglu S, Bolkent S. Exendin-4 attenuates renal tubular injury by decreasing oxidative stress and inflammation in streptozotocin-induced diabetic mice. Growth Factors. 2015; 33(5-6): 419-29.

139. Botfield HF, Uldall MS, Westgate CSJ, et al. A glucagon-like peptide-1 receptor agonist reduces intracranial pressure in a rat model of hydrocephalus. Sci Transl Med. 2017; 9(404).

140. Zhao Y, Li D, Zhao J, et al. The role of the low-density lipoprotein receptor-related protein 1 (LRP-1) in regulating blood-brain barrier integrity. Rev Neurosci. 2016; 27(6): 623-34.

141. Li H, Wang P, Huang F, et al. Astragaloside IV protects blood-brain barrier integrity from LPS-induced disruption via activating Nrf2 antioxidant signaling pathway in mice. Toxicology and Applied Pharmacology. 2018; 340: 58-66.

142. Hoyles L, Snelling T, Umlai UK, et al. Microbiome-host systems interactions: protective effects of propionate upon the blood-brain barrier. Microbiome. 2018; 6(1): 55.

143. Silverberg GD, Messier AA, Miller MC, et al. Amyloid efflux transporter expression at the blood-brain barrier declines in normal aging. Journal of Neuropathology and Experimental Neurology. 2010; 69(10): 1034-43.

144. Shibata M, Yamada S, Kumar SR, et al. Clearance of Alzheimer's amyloid-ss(1-40) peptide from brain by LDL receptor-related protein-1 at the blood-brain barrier. The Journal of Clinical Investigation. 2000; 106(12): 1489-99.

145. Erickson MA, Hansen K, Banks WA. Inflammation-induced dysfunction of the low-density lipoprotein receptor-related protein-1 at the blood-brain barrier: protection by the antioxidant $\mathrm{N}$-acetylcysteine. Brain Behav Immun. 2012; 26(7): 1085-94.

146. Knuckey NW, Palm D, Primiano M, et al. N-acetylcysteine enhances hippocampal neuronal survival after transient forebrain ischemia in rats. Stroke; A Journal of Cerebral Circulation. 1995; 26(2): 305-10 discussion 11

147. Patel P, Shah J. Role of Vitamin D in amyloid clearance via LRP-1 upregulation in Alzheimer's disease: A potential therapeutic target? Journal of Chemical Neuroanatomy. 2017; 85: 36-42.

148. Yang S, Krug SM, Heitmann J, et al. Analgesic drug delivery via recombinant tissue plasminogen activator and microRNA-183-triggered opening of the blood-nerve barrier. Biomaterials. 2016; 82: 20-33.

149. Fujiyoshi M, Tachikawa M, Ohtsuki S, et al. Amyloid- $\beta$ peptide(1-40) elimination from cerebrospinal fluid involves low-density lipoprotein receptor-related protein 1 at the blood-cerebrospinal fluid barrier. Journal of Neurochemistry. 2011; 118(3): 407-15

150. Wu XH, Pang YX, Qiu HY, et al. Effects of subchronic aluminum lactate exposure on learning and memory and transportation of $A \beta$ in bloodcerebrospinal fluid in rats. Zhonghua Lao Dong Wei Sheng Zhi Ye Bing Za Zhi. 2016; 34(2): 90-4.

151. Pascale CL, Miller MC, Chiu C, et al. Amyloid-beta transporter expression at the blood-CSF barrier is age-dependent. Fluids Barriers CNS. 2011; 8: 21.

152. Stopa EG, Tanis KQ, Miller MC, et al. Comparative transcriptomics of choroid plexus in Alzheimer's disease, frontotemporal dementia and Huntington's disease: Implications for CSF Homeostasis. Fluids and Barriers of the CNS. 2018;15(1):18.

153. González-Marrero I, Giménez-Llort L, Johanson CE, et al. Choroid plexus dysfunction impairs beta-amyloid clearance in a triple transgenic mouse model of Alzheimer's disease. Front Cell Neurosci. 2015; 9: 17.

154. Rago B, Tierney B, Rodrigues A, et al. A multiplex HRMS assay for quantifying selected human plasma bile acids as candidate OATP biomarkers Bioanalysis. 2018; 10(9): 645-57. 
155. Zibara K, Zein NE, Sabra M, et al. Thyroxine (T(4)) transfer from blood to cerebrospinal fluid in sheep isolated perfused choroid plexus: Role of multidrug resistance-associated proteins and organic anion transporting polypeptides. Front Neurol. 2017; 8: 214.

156. Aly AE, Harmon BT, Padegimas L, et al. Intranasal delivery of
pGDNF DNA nanoparticles provides neuroprotection in the rat 6-hydroxydopamine model of Parkinson's disease. Mol Neurobiol. 2018; May 19.

157. Reinhold AK, Rittner HL. Barrier function in the peripheral and central nervous system- A review. Pflugers Arch. 2017; 469(1): 123-34. 